\title{
Collimation-induced experimental background studies at the CERN Large Hadron Collider
}

\author{
R. Bruce,,${ }^{1,}$ M. Huhtinen, ${ }^{1}$ A. Manousos,${ }^{1,2,3}$ F. Cerutti, ${ }^{1}$ L. Esposito, ${ }^{1}$ R. Kwee-Hinzmann, ${ }^{4}$ \\ A. Lechner, ${ }^{1}$ A. Mereghetti, ${ }^{1}$ D. Mirarchi, ${ }^{1}$ S. Redaelli, ${ }^{1}$ and B. Salvachua ${ }^{1}$ \\ ${ }^{1}$ CERN, 1211 Geneva, Switzerland \\ ${ }^{2}$ University of Innsbruck, 6020 Innsbruck, Austria \\ ${ }^{3}$ Aristotle University of Thessaloniki, 54124 Thessaloniki, Greece \\ ${ }^{4}$ John Adams Institute at Royal Holloway University of London, Egham TW20 OEX, United Kingdom
}

(Received 26 October 2018; published 26 February 2019)

\begin{abstract}
The data produced at the particle physics experiments at the Large Hadron Collider (LHC) contain not only the signals from the collisions, but also a background component from proton losses around the accelerator. Understanding, identifying and possibly mitigating this machine-induced background is essential for an efficient data taking, especially for some new physics searches. Among the sources of background are hadronic and electromagnetic showers from proton losses on nearby collimators due to beam-halo cleaning. In this article, the first dedicated LHC measurements of this type of background are presented. Controlled losses of a low-intensity beam on collimators were induced, while monitoring the backgrounds in the ATLAS detector. The results show a clear correlation between the experimental backgrounds and the setting of the tertiary collimators (TCTs). Furthermore, the results are used to show that during normal LHC physics operation the beam halo contributes to the total beam-induced background at the level of a percent or less. A second measurement, where the collimator positions are tightened during physics operation, confirms this finding by setting a limit of about $10 \%$ to the contribution from all losses on the TCTs, i.e. the sum of beam halo and elastic beam-gas scattering around the ring. Dedicated simulations of the halo-related background are presented and good agreement with data is demonstrated. These simulations provide information about features that are not experimentally accessible, like correlations between backgrounds and the distributions of proton impacts on the collimators. The results provide vital information about the dependence between background and collimator settings, which is of central importance when optimizing the LHC optics for maximum peak luminosity.
\end{abstract}

DOI: 10.1103/PhysRevAccelBeams.22.021004

\section{INTRODUCTION}

The Large Hadron Collider (LHC) [1,2] is a $27 \mathrm{~km}$ synchrotron, designed to collide two counterrotating beams of, up to, $7 \mathrm{TeV}$ protons or heavy ions of equivalent magnetic rigidity, inside four experimental detectors. The experiments are located at interaction regions (IRs) in four out of the eight straight sections of the ring. The geometry of the LHC is schematically illustrated in Fig. 1.

At the nominal luminosity of $10^{34} \mathrm{~cm}^{-2} \mathrm{~s}^{-1}$ the LHC produces about $8 \times 10^{8}$ inelastic proton-proton collisions per second at the low- $\beta^{*}$ [4] interaction points (IPs) inside the ATLAS [5] and CMS [6] detectors. Only a small fraction of these events include interesting physics

*roderik.bruce@cern.ch

Published by the American Physical Society under the terms of the Creative Commons Attribution 4.0 International license. Further distribution of this work must maintain attribution to the author(s) and the published article's title, journal citation, and DOI. processes, the remainder constituting the dominant experimental background. Backgrounds with much lower intensity, but very different characteristics, arise from losses of beam protons in the vicinity of the experiments [7]. The collisions of protons with residual gas molecules or machine elements induce particle showers which can reach the detectors and constitute machine-induced backgrounds [8-16]. Since the LHC detectors are optimized to efficiently detect and measure particles emerging from collisions at the IP, they have, in their central regions, high granularity in the longitudinal $(z)$ and the azimuthal $(\phi)$ direction. The particle tracks originating from beam losses are usually almost parallel to the beam and, unlike particles from the IP, can traverse a large number of detector cells along $z$. This can result in high occupancy and thereby degrade the data-taking efficiency. High-energy muons are a particularly important component of the machine-induced background, since they can lose a major fraction of their energy by radiative processes in the calorimeters of the detectors. If such losses are large enough they can get reconstructed as jets, which are important objects in most 
physics analyses. Such erroneously reconstructed jets are referred to as fake jets. If overlaid with a collision event, they can lead to missing transverse energy which might be confused with some signatures of rare new physics [17-20]. Therefore a good understanding and minimization of machine-induced backgrounds is among the requirements to reach optimal experimental conditions.

Machine-induced backgrounds arise from three conceptually different sources: (i) inelastic collisions of the beam protons with residual gas in the LHC beam vacuum; (ii) beam-halo losses on limiting apertures close to the experiment; and (iii) elastic beam-gas scattering around the accelerator, out of which a fraction results in protons lost on apertures close to the experiment. Operational experience with the LHC, so far, suggests that inelastic beam-gas interactions constitute the dominant source of machine-induced backgrounds [21,22]. This article, however, focuses on a first quantitative measurement of the halorelated background component during $6.5 \mathrm{TeV}$ proton operation. Such a study must be done under dedicated beam conditions in order to have well-defined beam-halo losses and minimize the effect of other background components.

The beam halo is constantly populated by various processes, like elastic collisions at the experiments and with residual gas, noise in the rf system and the feedback, intrabeam scattering, resonances and instabilities. For this study beam-loss measurements under special conditions described in Ref. [23] are combined with ATLAS data, published in Ref. [24]. The LHC beam cleaning system is described in Sec. II and the measurement procedure in Sec. III. The results, discussed in Sec. IV, are used in Sec. V to make a quantitative assessment of the magnitude of beam-halo background during physics operation, which in turn provides important inputs for decisions on the future machine configuration. A second experimental test, aimed to set a rough limit on the background from elastic beam-gas scattering, is described in Sec. VI. Finally, the measured backgrounds are compared with simulations in Sec. VII. All abbreviations used in the text are summarized in the Appendix.

\section{LHC BEAM CLEANING}

The collimators of the betatron cleaning insertion in interaction region 7 (IR7) form the backbone of the multistage LHC collimation system [25-30]. All transverse halo particles with high betatron amplitudes should first hit a primary collimator (TCP) in IR7, installed in the horizontal, vertical, and skew planes. Most protons that scatter out of the TCPs are intercepted by secondary collimators (TCSGs) and other collimator families at larger openings. Particles leaking out of the TCSGs constitute tertiary halo, which eventually could end up on the active absorbers (TCLA) in IR7 or the tertiary collimators (TCTs) $150 \mathrm{~m}$ upstream of the experiments. A similar cleaning hierarchy consisting of TCP, TCSGs, and TCLAs is installed for momentum cleaning in IR3, and special protection collimators (TCDQ and TCSP) are installed in IR6 to protect the machine in case of beam extraction failures. The TCPs and TCSGs are 0.6 and $1 \mathrm{~m}$ long, respectively. Both are made of carbon fiber composite, while the $1 \mathrm{~m}$ long TCTs and TCLAs are made of tungsten. All these collimators consist of two movable parallel jaws with the beam passing between them.

Each TCT assembly includes one horizontal (TCTH) and one vertical (TCTV) set of jaws. Their primary role is to protect the magnets of the final focusing system but they also intercept the tertiary halo and some of the showers from upstream beam-gas interactions. When halo particles interact with the material of a TCT, hadronic and electromagnetic showers are induced and some secondary particles leak through or scatter out of the TCT. Most of them are stopped in downstream magnetic elements or shielding, but a small fraction reaches the experiments.

This study is focused on background in the ATLAS experiment due to beam protons lost in IR7. It is evident from Fig. 1 that this creates an asymmetry between the clockwise B1 and counterclockwise B2: while the distance for B1 is only two octants, B2 has to travel six octants with several tight apertures (IR6, IR5 and IR3) along its path.

The intensity of the beam-halo background depends on the beam conditions and the machine configuration, in particular the collimator settings. In general the beam-halo background is reduced if the upstream collimators are set at tight apertures such that the tertiary halo is minimized.

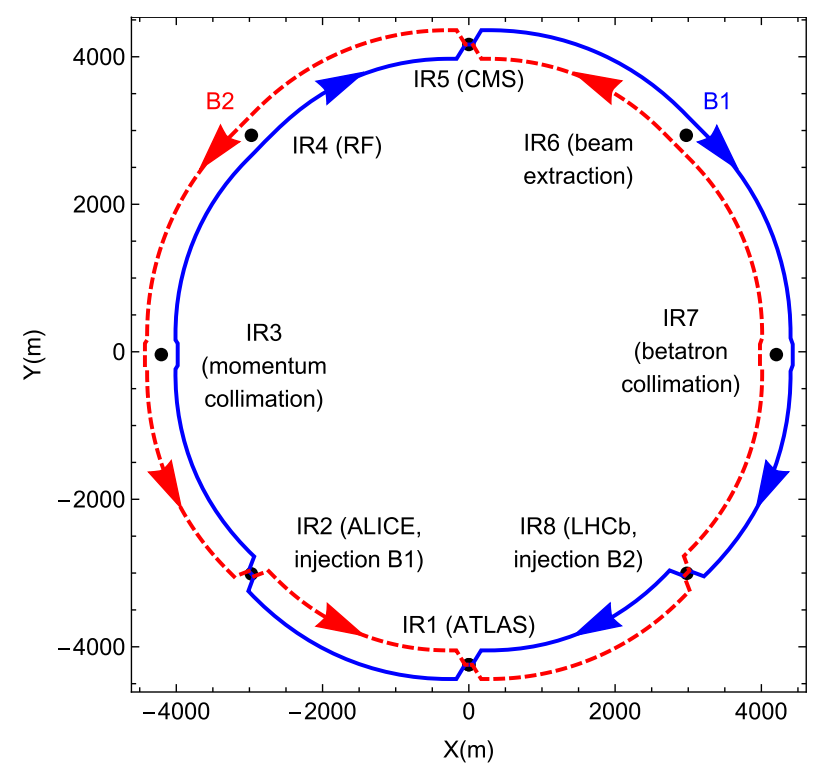

FIG. 1. The schematic layout of the LHC showing the eight insertions of which four house experiments. Of particular importance for this study is the betatron cleaning in IR7. The two counterrotating beams are shown schematically, i.e. their actual separation of $194 \mathrm{~mm}$ in the arcs is not to scale. This figure was adapted from Ref. [3]. 
It can, however, also depend on the beam optics, in particular the dispersion and the betatron phase advances between IR7 and the TCT. For fixed collimation and optics settings, the beam-halo background is directly proportional to the instantaneous loss rate of halo protons on the TCPs.

Beam-halo backgrounds at the LHC have been studied in simulations during the design stage for the nominal $7 \mathrm{TeV}$ configuration [7-15] and later for the first LHC run at 3.5-4 TeV [16,31] and for the foreseen LHC luminosity upgrade [31-33]. Measurements of machine-induced backgrounds have been reported by ATLAS [21,22], but it is difficult to disentangle the various background contributions during standard physics operation, when all sources are superimposed. However, data recorded during a short special physics run in 2012 with low intensity and high $\beta^{*}$ revealed that sufficiently large beam losses in the cleaning insertions can be seen as increased backgrounds in ATLAS [22]. This suggested that a direct measurement of the beamhalo related background, with normal low- $\beta^{*}$ optics, might be feasible under some special conditions.

Even though there is strong evidence [21,22] that the machine-induced backgrounds are dominated by beam-gas collisions, a quantification of the beam-halo component is important for optimization of the LHC performance. Since the beam-halo background is expected to increase with tighter TCT gaps, there could be a lower limit on the TCT setting below which the background is not deemed acceptable. This in turn limits the normalized aperture that can be protected in the superconducting quadrupole magnets of the final focusing system. Therefore the amount of beamhalo background, and how much of it is acceptable, imposes constraints on the allowed $\beta^{*}$ and hence also on the achievable peak luminosity $[3,34]$.

\section{MEASUREMENT PROCEDURE IN LOSS-MAP TESTS}

Before high-intensity beams are allowed in the LHC, the protection and cleaning performance of the collimation system must be qualified. This is done in dedicated lossmap measurements [29,35-38], where controlled beam losses are provoked on a safe beam of low intensity, while recording the loss pattern around the LHC using beam loss monitors (BLMs) $[39,40]$. The BLMs are ionization chambers, attached to, e.g., magnets and collimators. Almost 4000 BLMs are installed around the LHC and, for all analyses presented in this article, the integrated signal over $1.3 \mathrm{~s}$ recorded at $1 \mathrm{~Hz}$ [41] is used. Any individual BLM measures the time distribution of losses at its location, but since shower development in the machine elements, and the location of the BLMs with respect to these elements, differ, two BLMs cannot be directly compared to determine the ratio of losses on two different accelerator elements. The ratio between signals from any two BLMs, however, can be used to determine how loss patterns change as a function of time or collimator settings.
Losses, either in the horizontal or vertical plane, are provoked on individual bunches by applying a whitenoise excitation with the transverse damper (ADT) [42] system. During the ADT excitation, with a typical duration of a few seconds, a large fraction of the affected bunch is lost on the TCP associated to the plane of excitation. The loss rates reach values of the order of $10^{9} \mathrm{~Hz}$ per bunch. In these conditions it is expected that the signals seen by the experiments are dominated by the showers from the TCT impacts, caused by the leakage out of IR7. Thus the loss maps provide a very clean environment for studying the beam-halo component of the background.

Normally, the loss-map measurements are performed in the accelerator while experiments are in the idle state. The new feature of the present study is that the ATLAS experiment was partially turned on in order to record background data [24] during the loss-map tests. The principal background monitoring methods used by ATLAS are based on a dedicated beam condition monitor (BCM) [43] to measure near-beam backgrounds and a monitoring of fake-jet rates in the calorimeters. Both of these methods rely on the availability of a few noncolliding bunches in the LHC bunch pattern. Most of the bunches used in the loss-map measurements are of this type, i.e. do not have a partner in the other beam to collide with at the ATLAS IP.

The $\mathrm{BCM}$ detector, with subnanosecond time resolution, consists of four diamond modules on each side of the IP. Two modules are in the vertical and two in the horizontal plane at a mean radius of $5.5 \mathrm{~cm}$ from the beam line and at a distance $|z|=184 \mathrm{~cm}$ from the IP. The BCM background trigger is based on the assumption that the background arrives in time with the proton bunch. A background count is provided if any module on one side has an early hit while any module on the other side has an in-time hit during the same bunch passage.

The ATLAS calorimeter system consists of several subdetectors. In the central barrel part they extend from a radius $r=1.5 \mathrm{~m}$ to $r=4.3 \mathrm{~m}$ and have a length of $\pm 3 \mathrm{~m}$ (electromagnetic) and $\pm 6 \mathrm{~m}$ (hadronic). The end cap calorimeters extend the coverage to almost $4 \pi$ around the IP [5]. The fake-jet monitoring is based on a single jet trigger with a low transverse momentum $\left(p_{\mathrm{T}}\right)$ threshold of $12 \mathrm{GeV}$. For the analysis presented in Ref. [24], which also describes in detail the cuts applied to this trigger, the events recorded were analyzed in order to extract the exact time, apparent $p_{\mathrm{T}}$ and position of each fake jet.

The $\mathrm{BCM}$ is designed to probe showers developing just outside of the beam pipe and is therefore most sensitive to local losses, although simulations indicate that it maintains some sensitivity at least up to the TCT location [44]. The fake jets in the calorimeters, especially the barrel part, are almost exclusively due to radiative energy losses of highenergy muons. Besides cosmic rays, these muons originate from beam losses far upstream. Since muons produced in 
interactions of $6.5 \mathrm{TeV}$ protons have a strong forward boost, they will not reach the radii of the barrel calorimeters $(r>150 \mathrm{~cm})$ unless they are produced at a large distance or deflected by the magnets of the LHC lattice. In this study the background originates from the TCT at $|z| \approx 150 \mathrm{~m}$ and the only dipole magnet traversed is the separation dipole D1, which extends from $|z|=59$ to $|z|=83 \mathrm{~m}$ and has a field of $1.2 \mathrm{~T}$.

Qualification loss-map tests in the LHC on two occasions were used for the measurements reported here. The first set of data was obtained during a test which took place on July 4, 2015, using the standard optics for physics of that year with $\beta^{*}=80 \mathrm{~cm}$ and a half crossing angle of $-145 \mu \mathrm{rad}$. A more extensive dataset was collected during a dedicated test on August 28, 2015, at $\beta^{*}=40 \mathrm{~cm}$ and zero crossing angle. In both cases the energy of the proton beams was $6.5 \mathrm{TeV}$.

During the $\beta^{*}=80 \mathrm{~cm}$ test, all collimators were kept at their standard physics operation settings, shown in Table I. Four pilot bunches, of $10^{10}$ protons each, and two nominal bunches, of around $10^{11}$ protons per bunch, were injected in each ring in order to have a filling scheme with nominal bunches colliding at all IRs [45]. All pilot bunches, but only one nominal bunch per beam, were noncolliding in ATLAS and could be used for the background measurement. Loss maps were performed by exciting sequentially bunches in B1 and B2 in either the horizontal or the vertical planes. Each bunch was used only in one plane.

During the $\beta^{*}=40 \mathrm{~cm}$ test, the openings of all TCTs simultaneously were varied between $7.8 \sigma$ and $10.3 \sigma$ in steps of $0.5 \sigma$ [46], while keeping the rest of the collimators at constant openings as shown in the middle column of Table I. At each TCT setting, a set of the four standard loss maps was performed (both beams and both planes), which

TABLE I. Collimator half gaps used during the tests described in this article, given in units of beam standard deviation $\sigma$, assuming the nominal $\beta$-function and a normalized emittance of $3.5 \mu \mathrm{m}$. The settings at $\beta^{*}=80 \mathrm{~cm}$ are the standard settings during physics operation in 2015, which were also used during the loss-map tests. At $\beta^{*}=40 \mathrm{~cm}$ the settings used in the 2015 loss-map test and the 2016 operational settings are both shown. The latter were used during the TCT closure test, discussed in Sec. VI.

\begin{tabular}{lccc}
\hline \hline $\begin{array}{l}\text { Collimator } \\
\text { family }\end{array}$ & $\beta^{*}=80 \mathrm{~cm}$ & $\begin{array}{c}\beta^{*}=40 \mathrm{~cm} \\
\text { (loss maps) }\end{array}$ & $\begin{array}{c}\beta^{*}=40 \mathrm{~cm} \\
(2016 \text { operation) }\end{array}$ \\
\hline TCP IR7 & 5.5 & 5.5 & 5.5 \\
TCSG IR7 & 8.0 & 7.5 & 7.5 \\
TCLA IR7 & 14.0 & 14.0 & 11.0 \\
TCP IR3 & 15.0 & 15.0 & 15.0 \\
TCSG IR3 & 18.0 & 18.0 & 18.0 \\
TCLA IR3 & 20.0 & 20.0 & 20.0 \\
TCT IR1,5 & 13.7 & $7.8-10.3$ & 9.0 \\
TCSP IR6 & 9.1 & 8.6 & 8.3 \\
TCDQ IR6 & 9.1 & 8.6 & 8.3 \\
\hline \hline
\end{tabular}

allows studying the dependence of background on the TCT setting.

For the test at $\beta^{*}=40 \mathrm{~cm}$, one nominal and 15 pilot bunches were injected into each LHC ring. Because the total intensity in loss-map tests is limited by machine protection, subdividing the beam into a large number of pilot bunches allowed performing measurements at different TCT settings within the same fill. In order to create losses over a longer time period for increased resolution, the nominal bunches were also excited. These excitations were done at a TCT setting of $8.8 \sigma$ in the vertical and horizontal plane for B1 and B2, respectively.

\section{MEASUREMENT RESULTS OF LOSS-MAP TESTS}

A typical loss distribution, around the LHC ring, resulting from an ADTexcitation at $\beta^{*}=40 \mathrm{~cm}$ with the TCTs at $8.8 \sigma$ is shown in Fig. 2. The main loss locations are found on the primary collimators in IR7, and the loss levels then decrease on downstream collimators in IR7. The losses on the TCTs, seen as black spikes in IR1 and IR5, are about 3 orders of magnitude below those in IR7.

The BLM signal at the TCPs in IR7 is very well resolved, being several orders of magnitude above the background noise. For monitors with lower signals the subtraction of pedestal noise of the electronics is mandatory, in particular for some monitors that constantly show higher rates than others. In order to remove the pedestal noise the count rate of each BLM is averaged over a period shortly before the start of the loss-map test, i.e. in absence of any excitation,

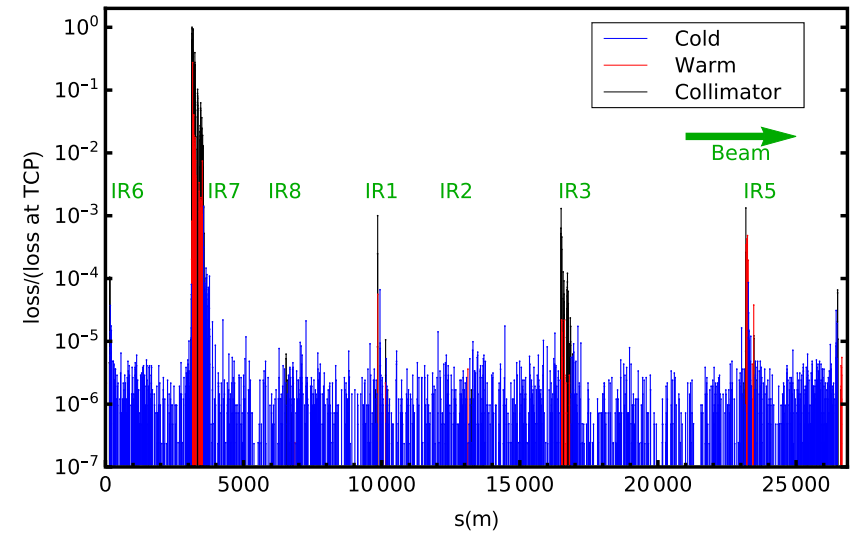

FIG. 2. Beam loss distribution around the LHC, measured with the BLMs using a $1.3 \mathrm{~s}$ integration time. The initial loss is provoked by a white-noise excitation of the ADT in the vertical plane of B1 at $6.5 \mathrm{TeV}$ beam energy and $\beta^{*}=40 \mathrm{~cm}$. The TCTs were positioned at $8.8 \sigma$ and the rest of the collimator settings are shown in Table I. The background noise of the electronics, taken as the BLM signals without any excitation, has been subtracted. The color indicates if the monitor is attached to a cold machine element (blue), a warm machine element (red), or a collimator (black). 

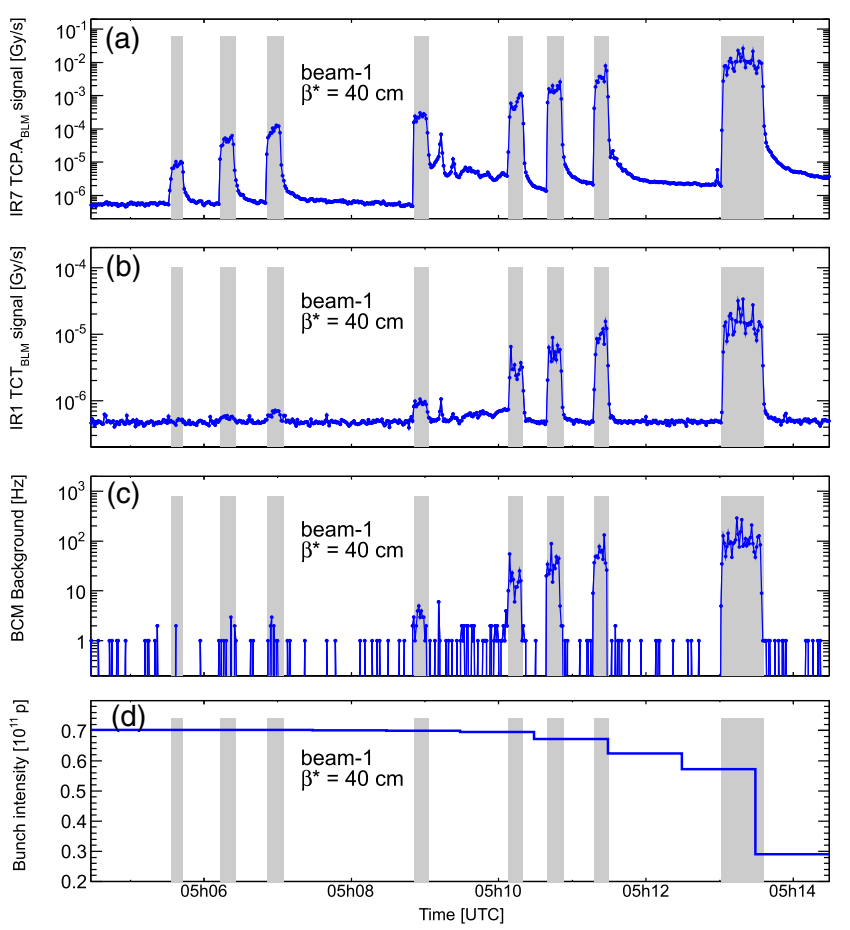

FIG. 3. Various observables during consecutive vertical excitations of a nominal bunch in B1 at $\beta^{*}=40 \mathrm{~cm}$ with the TCT at $8.8 \sigma$. The uppermost plot shows the TCP.A BLM signal, the second plot from the top shows the sum of the BLM signals at IR1 TCTH and TCTV, the third plot shows the measured BCM background in ATLAS from Ref. [24], and the bottom plot the intensity of the affected bunch. The shaded areas indicate the time limits of the excitations.

and subtracted from the BLM measurement during the excitation.

Figure 3(a) shows a typical example of measured BLM signals at the TCP in IR7 in a period when eight consecutive excitations with increasing amplitude were performed on a nominal bunch. The loss is measured by a BLM called TCP.A, which is placed about $6 \mathrm{~m}$ downstream of the vertical TCP and $4 \mathrm{~m}$ downstream of the horizontal one. Therefore it intercepts the showers due to both, horizontal and vertical, excitations, all of which are reflected as clearly identifiable bumps in the BLM data. The bump limits, shown as shaded vertical bands, are determined from the rising and falling edges. Figure 3(b) shows the loss measurements at IR1 TCTs, where the sum of two BLMs, associated to the TCTH and TCTV are used. For the smaller losses, at the left in the plot, the bumps are barely distinguishable from the background. The signal is extracted as a sum between the bump limits, determined from the TCP.A BLM data. The pedestal, fitted in an excitation-free area, is subtracted. Figure 3(c) shows the BCM background measured by ATLAS. Like for the TCT BLMs, the signal is taken as the sum over the shaded areas after subtracting the pedestal. As the excitation strength increases, and the loss rate on the TCP goes up, the TCT
BLM and the ATLAS BCM eventually reach signals up to 2 orders of magnitude above the pedestal, which for the $\mathrm{BCM}$ is mostly due to beam-gas interactions. A sum over the pedestal indicates that the BCM measures a beam-gas background at the level of less than $0.5 \mathrm{~Hz}$ per nominal bunch. It is therefore clear that during the stronger excitations, both the TCT BLM signals and BCM background are fully dominated by the beam-halo losses and all other background sources are negligible. Figure 3(d) shows the intensity of the excited bunch as a function of time. The coarse resolution arises from the fact that the intensities of individual bunches were logged only once per minute. Nevertheless, it can be seen that the amount of intensity loss correlates with the size of the loss bump at the TCPs.

Figure 4 shows, as a function of the TCT opening for the various excitations during the test, the sum of the signals
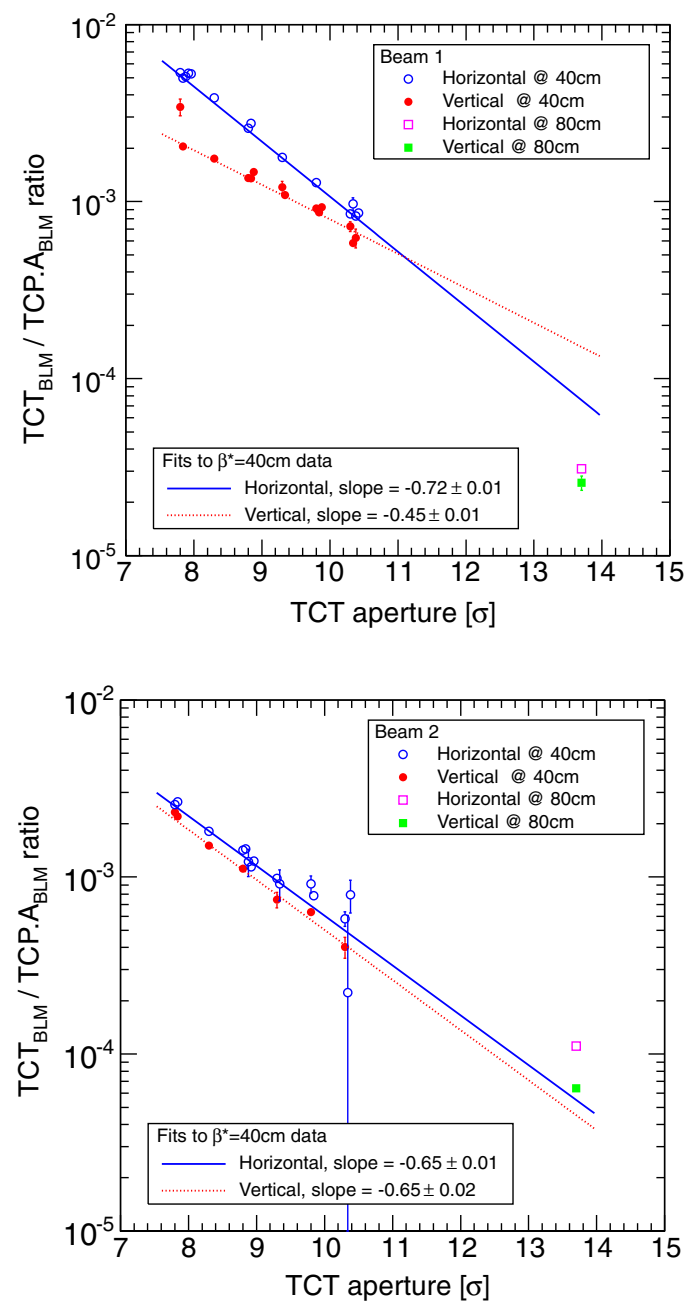

FIG. 4. Ratio of signals from the BLMs of the IR1 TCTs to the IR7 TCPs as a function of TCT opening for B1 (top) and B2 (bottom), combining the tests at $\beta^{*}=40 \mathrm{~cm}$ and $\beta^{*}=80 \mathrm{~cm}$. For enhanced visibility, the points are slightly shifted with respect to each other to better separate the error bars. The fits do not take into account the $\beta^{*}=80 \mathrm{~cm}$ data, i.e. the values at a TCT setting of $13.7 \sigma$. 
from the BLMs associated to the horizontal and vertical TCTs of IR1 normalized by the measurement of the TCP.A BLM. For a given plane, the latter is proportional to the amount of beam lost, which can vary by a significant factor between excitations. The ratio between the TCT BLM signals and the TCP.A BLM, however, is proportional to the fraction of halo leaking from IR7 to the IR1 TCTs and any dependence on excitation strength and original amount of beam lost should be removed. The small spread between the points for the same excitation plane and at the same TCT setting supports this assumption.

The error bars in Fig. 4 have been estimated by calculating the TCT/TCP ratio for each $1 \mathrm{~s}$ time bin during the excitation separately and determining the average and its rms from these per-bin ratios. This method covers uncertainties from counting statistics and other effects that might alter the ratio during the peak. However, it does not account for systematic uncertainties like a possible dependence of the BLM response on impact distribution or possible changes, like orbit drifts, that occur between different excitations.

Results are presented for both beams and planes and for both the $\beta^{*}=40 \mathrm{~cm}$ and $\beta^{*}=80 \mathrm{~cm}$ tests. As expected, the TCT losses increase strongly with decreasing opening. If the TCT opening is wider a larger number of protons will miss it and be intercepted in IR7 or in other IRs on the next turn. The lines shown in Fig. 4 indicate that, to a good approximation, the ratio, as a function of TCT opening, can be fitted with an exponential, which indicates that the transverse halo distribution falls off exponentially with increasing amplitude. The points at $\beta^{*}=80 \mathrm{~cm}$ do not exactly match the fit, but this can be explained by the different optics and slightly different collimator settings in IR6 and IR7, as detailed in Table I.
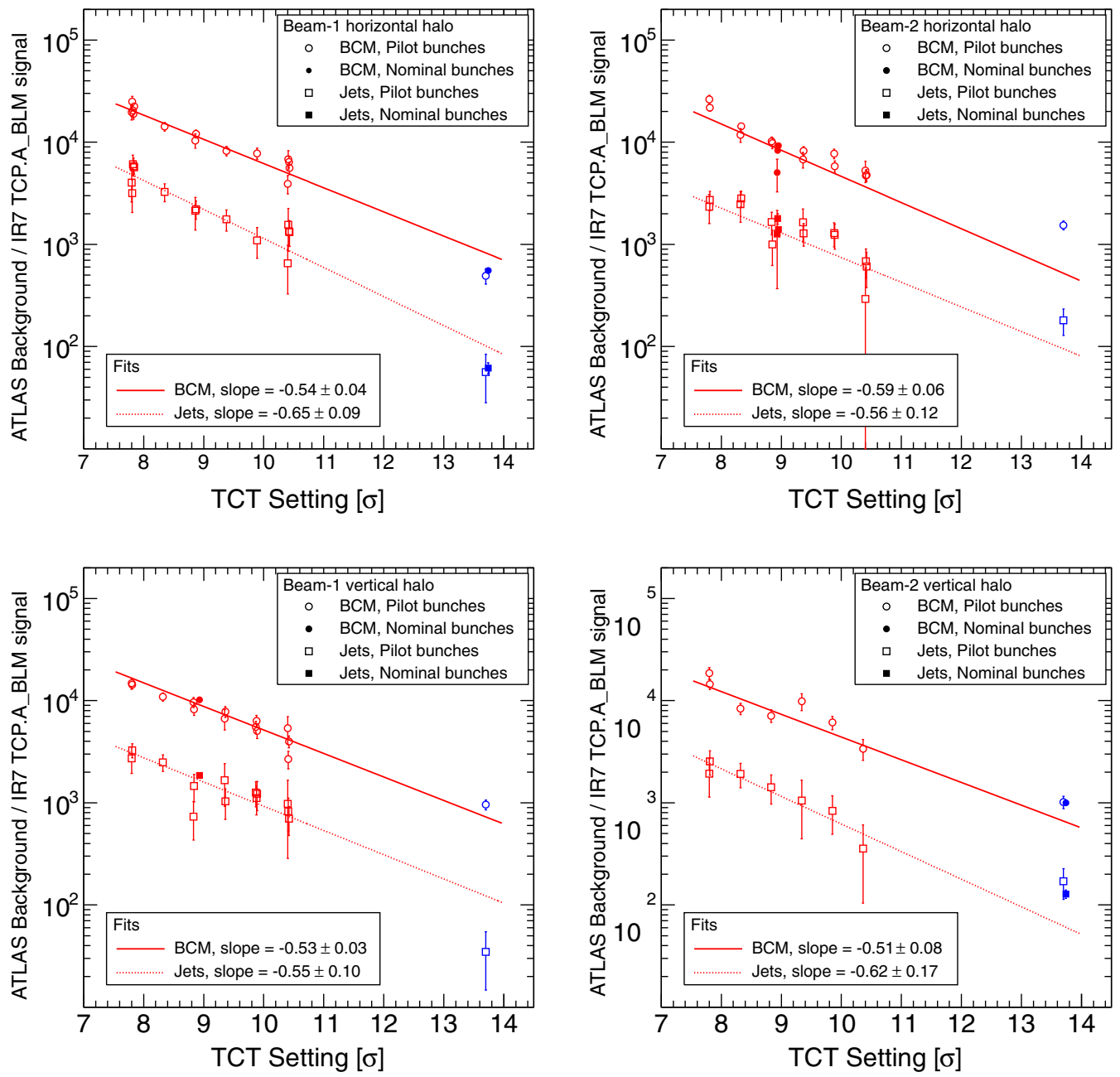

FIG. 5. Ratio between background rates measured by ATLAS and the signal from the TCP BLMs in IR7 for losses provoked on B1 (left) and B2 (right) in the horizontal (top) and vertical (bottom) planes. The red symbols correspond to the $\beta^{*}=40 \mathrm{~cm}$ test with TCT settings varied from 7.8 to $10.3 \sigma$. The blue points at $13.7 \sigma$ correspond to the $\beta^{*}=80 \mathrm{~cm}$ test. When several measurements fall on the same TCT setting, the points are slightly shifted in the plot in order to separate their error bars. The lines show exponential fits to the $\beta^{*}=40 \mathrm{~cm}$ points, of the form $C e^{(s \times A)}$, where $A$ is the TCT aperture. The ATLAS data are taken from Ref. [24]. 
Since the halo-related background in the experiments is assumed to originate from losses on the TCTs, the ratio between the ATLAS background measurement and the loss rate on the TCTs should be constant. In Ref. [24] it is shown that, within the uncertainties, this is the case for the $\beta^{*}=40 \mathrm{~cm}$ data, while the $\beta^{*}=80 \mathrm{~cm}$ data shows some deviation, presumably due to the different optics at the IP. Consequently the ratio between the ATLAS background and the TCP.A BLM signal, as a function of the TCT setting, should be fitted by an exponential. Figure 5 shows these ratios for both, BCM and fake-jet backgrounds, during excitations in the two planes and for both beams. Although the data points have a slightly larger spread than in Fig. 4, the correlation between the aperture of the TCTs and the measured background is clearly evident for both, the BCM and the fake jets. The $\beta^{*}=40 \mathrm{~cm}$ data are well fitted by exponentials. The slopes $(s)$ of these fits are very similar to those in Fig. 4, which is consistent with the hypothesis that the background seen in the experiments is proportional to the rate of protons hitting the TCTs. The extrapolation of the fits to the $\beta^{*}=80 \mathrm{~cm}$ data points reveals that for $\mathrm{B} 1$ the agreement is rather good, although with a slight tendency for the fits to overshoot. For B2 the opposite is found, i.e. in both planes and both background observables the fits undershoot the data.

Since the BLM measurement is a running sum over $1.3 \mathrm{~s}$ with $1 \mathrm{~Hz}$ sampling frequency, it is not perfectly aligned with the ATLAS background data. Therefore the method of estimating the uncertainties from the bin-to-bin variation of the ratio is not applicable. Figure 3, however, shows that the signal in the TCP.A BLM is very clear with correspondingly small uncertainties so that the dominant uncertainty in the ratio must arise from the ATLAS background measurement. This motivates to take the counting statistics of the ATLAS measurement as the total uncertainty, shown as error bars in Fig. 5.

The background data can also be normalized by the number of protons lost on the TCPs instead of the TCP BLM signal. The intensity of each individual bunch is measured by the beam current transformer (BCT) and logged once per minute. Since the drop in intensity during the loss-map tests is almost entirely due to the excitationinduced losses in IR7 (see Fig. 3), it can be assumed that all protons lost from the beam hit on a TCP in IR7. The numbers of background counts per proton lost on the TCP are summarized in Table II for the two most important configurations, namely the 2015 operational scenario with $\beta^{*}=80 \mathrm{~cm}$ and a $13.7 \sigma$ TCT setting, and $\beta^{*}=40 \mathrm{~cm}$ with a $8.8 \sigma$ TCT, which is closest to 2016 operation. These were also the configurations where nominal bunches were used for the loss maps. In order to maximize the background signals in the experiments the larger intensity of these nominal bunches was fully utilized by prolonged excitations on each of them.
TABLE II. Ratio of background counts to the number of lost protons on the IR7 TCPs. Data, taken from Ref. [24], are shown for the ATLAS BCM and fake-jet counts in the ATLAS calorimeters, per lost proton on the IR7 TCPs. The shown results are for a TCT setting of $8.8 \sigma$ at $\beta^{*}=40 \mathrm{~cm}$ and $13.7 \sigma$ at $\beta^{*}=80 \mathrm{~cm}$. The results are the average over the excitations in the horizontal and vertical planes. The indicated error margins correspond to the uncertainty coming from the background counting rates.

\begin{tabular}{lccc}
\hline \hline Beam & $\beta^{*}(\mathrm{~cm})$ & BCM $($ counts/p) & Fake jets $($ counts/p) \\
\hline B1 & 40 & $(10.3 \pm 0.2) \times 10^{-8}$ & $(1.9 \pm 0.1) \times 10^{-8}$ \\
B2 & 40 & $(6.2 \pm 0.1) \times 10^{-8}$ & $(10.1 \pm 0.4) \times 10^{-9}$ \\
B1 & 80 & $(5.2 \pm 0.2) \times 10^{-9}$ & $(5.4 \pm 0.7) \times 10^{-10}$ \\
B2 & 80 & $(9.4 \pm 0.3) \times 10^{-9}$ & $(1.2 \pm 0.1) \times 10^{-9}$ \\
\hline \hline
\end{tabular}

The BCM counts per lost proton are a factor 5-10 higher than the fake-jet counts. This is not surprising, since these two background observables are based on totally different physics. While the BCM records any charged particle passing through its small sensors close to the beam line, the fake jets are created by high energy muons experiencing a large, and thus rare, radiative energy loss anywhere in the calorimeters.

\section{QUANTITATIVE ESTIMATES OF BEAM-HALO BACKGROUND IN PHYSICS OPERATION}

The loss-map measurements presented in Fig. 5 and Table II provide clean estimates of the amount of background seen by the experiments as a function of the beam losses on the TCPs. These results can be used to estimate the background rates in ATLAS during standard physics operation, by scaling them to the corresponding loss rate on the TCPs. The latter can be estimated for standard operation in 2015 and 2016 either from the bunch intensity data measured by the BCT or from the TCP BLM signals.

Neither of these two methods can provide a clean and direct measurement of the halo-related loss rate. The BCT measures the bunch intensity at percent level accuracy, but this measurement includes all loss processes. In the lossmap tests, when the excitation is turned on, all other processes are negligible compared to the halo losses. In a normal physics fill this is not the case. A large fraction of the beam intensity is lost due to luminosity production at the experiments while halo losses and beam-gas interactions are, at most, at the same level. During 2015 each bunch-bunch encounter in ATLAS resulted in 13.5 inelastic proton-proton collisions, averaged over all data taking. With a similar number for CMS and a revolution frequency of $11245 \mathrm{~Hz}$, each bunch lost, on average, about $3 \times 10^{5}$ protons per second due to luminosity. This luminosity component has to be subtracted from the total intensity loss measured by the BCT. Protons scattered elastically at the IP remain in the machine aperture. These and the remaining 
noncollisional losses are conservatively assumed to all hit the IR7 TCPs. This results in an overestimation, since the losses from inelastic beam-gas scattering occur around the entire ring and only a small amount of affected protons reach the collimators [16].

The signals of the TCP BLMs are proportional to local losses in IR7, however, they do not directly measure the number of lost protons, but rather the intensity of produced particle showers. The BLM measurement is also subject to a cross-talk effect: a shower generated by the loss on one TCP generates a signal also on BLMs associated to other downstream collimators. Therefore, to disentangle the losses between the different TCPs and to reconstruct the number of lost protons, a method described in Ref. [47] is used: the BLM signals are first recorded during loss maps with known excitation plane and intensity loss. The obtained results are then used to construct a matrix with the response of each BLM from each type of loss. The initial losses for any given BLM pattern can then be found through a single-value decomposition using the BLM response matrix. The total rate is estimated as the sum of horizontal and vertical losses.

Earlier studies have shown that the noncollisional loss rate is significantly higher during the first hour than later in the fill $[48,49]$. This suggests that the effective beam-halo background rates could evolve in the course of a long physics fill. Therefore, with both analysis methods, the average loss rates over two different time intervals are considered: the first hour of collisions, and the fifth hour.

The results are shown in Fig. 6 for all LHC physics fills which reached $5 \mathrm{~h}$ of length and the mean values over the years are presented in Table III.

As can be seen from Table III, there are significant differences between the two beams, the two years, and the two time windows within a fill. In 2016, the noncollisional losses are, on average, about a factor 5-10 lower in the fifth hour than in the first hour. Another striking feature is that in 2016 the losses in B1 during the first hour are about a factor of 2 higher than in B2. In 2015, the differences between the beams and the time windows are much smaller. The reason for the differences are not known in detail, but they could be related to imperfections on the feedback system or the magnetic lattice.

As detailed above, the BCT method provides a very accurate measurement of the total noncollisional losses, but it is not possible to determine exactly how large a fraction of these losses comes from impacts on the TCPs. For the BLM method, uncertainties come from possible differences in impact distribution between the losses at any given moment and the reference measurement where the BLM response matrix was constructed. By construction, in particular due to not subtracting the inelastic beam-gas contribution from the intensity loss, the BCT estimates should be higher. Table III, however, shows that differences are in both directions, but in all cases the methods agree
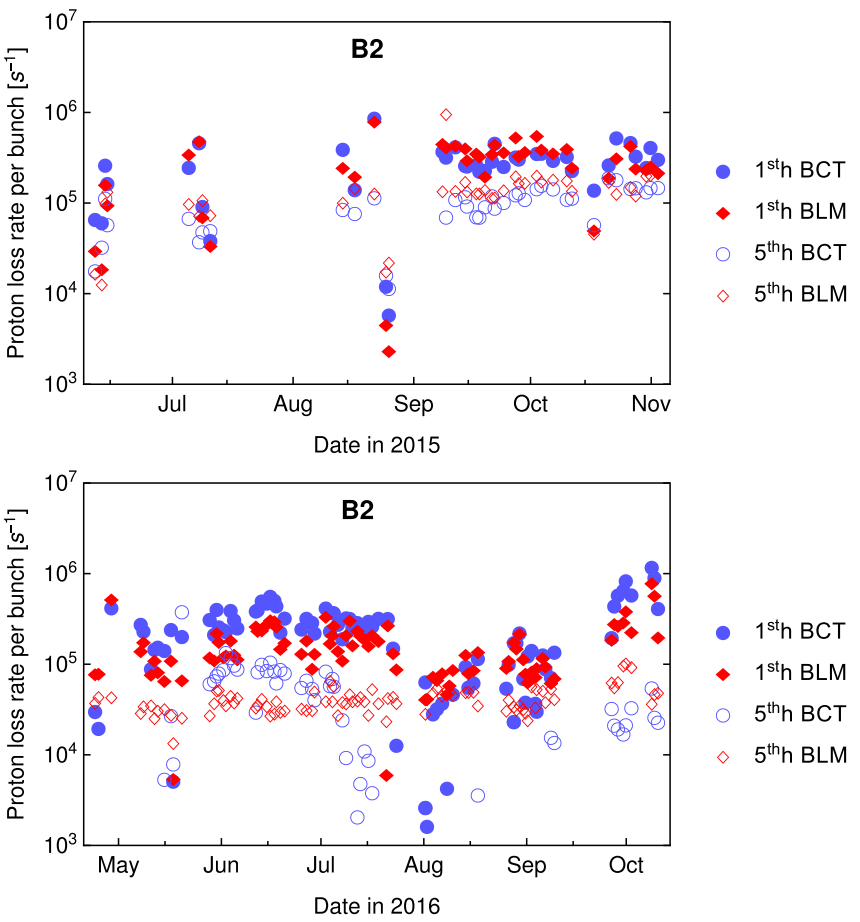

FIG. 6. Average beam loss rates per bunch of B1 and B2 on the TCPs as obtained from the BCT intensity data with the luminosity losses subtracted or from the IR7 BLMs. Results are shown for the two different time periods, the first and the fifth hour, of every physics fill in 2015 (with $\beta^{*}=80 \mathrm{~cm}$ ) and 2016 (with $\beta^{*}=40 \mathrm{~cm}$ ). 
TABLE III. Average beam loss rates, as obtained from the BLM and BCT data shown in Fig. 6, during physics operation in 2015 and 2016. Rates are given in units of protons/s/bunch for the first and fifth hour of collisions.

\begin{tabular}{|c|c|c|c|c|}
\hline \multirow[b]{2}{*}{ Time window } & \multicolumn{2}{|c|}{$2015, \mathrm{~B} 1$} & \multicolumn{2}{|c|}{ 2016, B1 } \\
\hline & BCT & BLM & BCT & BLM \\
\hline 1st hour & $3.6 \times 10^{5}$ & $2.6 \times 10^{5}$ & $4.9 \times 10^{5}$ & $5.2 \times 10^{5}$ \\
\hline \multirow[t]{2}{*}{5 th hour } & $1.1 \times 10^{5}$ & $0.7 \times 10^{5}$ & $0.5 \times 10^{5}$ & $0.7 \times 10^{5}$ \\
\hline & \multicolumn{2}{|c|}{$2015, \mathrm{~B} 2$} & \multicolumn{2}{|c|}{ 2016, B2 } \\
\hline Time window & BCT & BLM & $\mathrm{BCT}$ & BLM \\
\hline 1 st hour & $2.9 \times 10^{5}$ & $3.0 \times 10^{5}$ & $2.6 \times 10^{5}$ & $1.7 \times 10^{5}$ \\
\hline 5th hour & $1.0 \times 10^{5}$ & $1.5 \times 10^{5}$ & $0.5 \times 10^{5}$ & $0.4 \times 10^{5}$ \\
\hline
\end{tabular}

within $50 \%$. In view of the uncertainties involved, this can be considered a good agreement.

Using the calculated beam-loss rates and the background observed per lost proton on the TCPs, given in Table II, it is possible to estimate the absolute halo-related background rates in ATLAS during physics runs. In order to obtain an upper estimate, the loss rates from the BCT during the first hour of physics are used. The results for the BCM background and the fake jets are presented in Table IV, for both beams and years. The estimated halo contribution to the BCM background reaches up to $50 \mathrm{mHz} /$ bunch at $\beta^{*}=40 \mathrm{~cm}$, but amounts to only a few $\mathrm{mHz} /$ bunch at $\beta^{*}=80 \mathrm{~cm}$. The total BCM background rates in ATLAS during $2015\left(\beta^{*}=80 \mathrm{~cm}\right)$ operation are reported to be 3-5 Hz per bunch [24], which implies that the halo-related contribution is at the per-mil level. For the fake jets, Table IV indicates a beam-average rate of $0.3 \mathrm{mHz} /$ bunch at $\beta^{*}=80 \mathrm{~cm}$. A corresponding measurement by ATLAS for 2015 operation is not available, but Ref. [24] implies that this would be less than a percent of the total. In 2016, with a tighter TCT setting, the relative importance of the halorelated contribution increases, but still remains at a negligible level of about $1 \%$. If, instead of the first hour BCT results, the fifth hour BLM results would be used, the obtained rates would be lower by up to a factor of 7 .

TABLE IV. Estimated background count rates in ATLAS during physics operation in $\mathrm{Hz} /$ bunch, calculated as the product of the average loss rates on the TCPs during standard operation (taken from the BCT measurement for the first hour in Table III) and the background counts measured per proton lost on the TCP (Table II).

\begin{tabular}{lccccc}
\hline \hline & \multicolumn{2}{c}{$2015, \beta^{*}=80 \mathrm{~cm}$} & & \multicolumn{2}{c}{$2016, \beta^{*}=40 \mathrm{~cm}$} \\
\cline { 2 - 3 } \cline { 5 - 6 } Configuration & $\mathrm{B} 1$ & $\mathrm{~B} 2$ & & $\mathrm{~B} 1$ & $\mathrm{~B} 2$ \\
\hline BCM & $1.9 \times 10^{-3}$ & $2.7 \times 10^{-3}$ & & $4.9 \times 10^{-2}$ & $1.6 \times 10^{-2}$ \\
Fake jets & $1.9 \times 10^{-4}$ & $3.5 \times 10^{-4}$ & & $9.3 \times 10^{-3}$ & $2.6 \times 10^{-3}$ \\
\hline \hline
\end{tabular}

The preceding discussion highlights that the total uncertainty of the calculated background rates in Table IV is rather large. The uncertainty of the transfer function from the TCP losses to the ATLAS background is dominated by the counting statistics in the loss-map test and amounts to only a few percent. The dominant uncertainty, however, comes from the loss rate determination in a physics fill. The differences between the two independent methods, of up to $50 \%$, give a rough estimate on this uncertainty. The estimates in Table IV are already constructed as upper values in this respect. In addition, any differences in loss distribution on the TCPs in physics fills, compared to the loss-map measurements, could influence the result. Even if a combined uncertainty of a factor 2 would be assumed on these conservative estimates, the beam halo remains a negligible contribution to the total background also during the first hour of physics operation for both studied years.

The exponential fits in Fig. 5 indicate an average slope of $s=-0.5$. This implies that if the TCT is closed by one nominal $\sigma$, while all other settings remain the same, the halo-related component of the background increases by $60 \%$. However, for it to reach $10 \%$ of the total beam background, it would need to increase by a factor of about 10. A simple extrapolation of the fits shows that such a tenfold increase would require a TCT aperture of about $4.5 \sigma$. Obviously this extrapolation is not realistic, since such a setting would violate the collimation hierarchy, i.e. the TCTs would become primary collimators. In reality the TCTs can never be operated at a tighter setting in $\sigma$ than the TCSGs in IR7, which were at $7.5 \sigma$ in 2016 . These considerations lead to the conclusion that beam-halo backgrounds in the experiments do not impose a limit on reducing the TCT aperture, as long as the TCT respects its role in the multistage cleaning hierarchy.

\section{TOTAL BACKGROUND FROM LOSSES ON THE TCT IN PHYSICS OPERATION}

The results of Sec. V show that beam halo contributes by a negligible amount to the total machine-induced background in the experiments. The loss-map tests, however, do not probe the rate of protons ending up on the TCT as a result of elastic beam-gas scattering. In order to further quantify the effect of the TCT setting on the background, assessing the contributions from both beam halo and elastic beam-gas scattering, another experimental test was done by slightly reducing the TCT aperture in a normal physics fill.

The test was done on October 18, 2016, at the end of a 6.5 TeV physics fill with $\beta^{*}=40 \mathrm{~cm}, 2200$ nominal bunches per beam, and the collimator settings in the rightmost column of Table I. The transverse emittances were in the range $2-2.5 \mu \mathrm{m}$ and at the time of the test the average bunch intensity was around $8 \times 10^{10}$ protons. The total beam intensity ensured a representative dynamic pressure of the residual gas in the beam vacuum. 
Around $14 \mathrm{~h}$ after the beams were first brought into collision, the aperture of the vertical TCTs in IR1 and IR5 was reduced. Machine protection considerations, in particular the risk of asynchronous beam dumps, prevented a movement of the horizontal TCTs and imposed a limit of $400 \mu \mathrm{m}$ on the vertical movement. This corresponds to a reduction of the aperture from the nominal $9 \sigma$ to about $8.4 \sigma$. Both jaws of each vertical TCT were moved symmetrically in order to stay centered around the beam. The machine was then left with these tighter TCT settings for about $1.5 \mathrm{~h}$, while monitoring the experimental backgrounds. After this time, the TCTs were retracted again to the standard physics settings. The settings of the other collimators, which were not changed during this test, can be found in the last column of Table I.

The BCM backgrounds observed by ATLAS during this test [24] are shown in Fig. 7. No changes in measured background rates are visible during the time interval, shaded in gray, when the TCTs were tighter. The background evolution of both beams was fitted by a secondorder polynomial, using the data before and after the period of reduced TCT aperture. It is shown in Ref. [24] that an increase of more than $1 \%-2 \%$ of the background, averaged over the shaded area, could be resolved as a shift with respect to a corresponding time average over the fit. The results of Sec. IV suggest that a reduction of the TCT aperture by $0.6 \sigma$ would increase the halo-related background by about $30 \%$. However, since only the horizontal TCT was moved, the effect is probably only half of this. Even at $15 \%$ the effect still is a factor of 10 larger than the

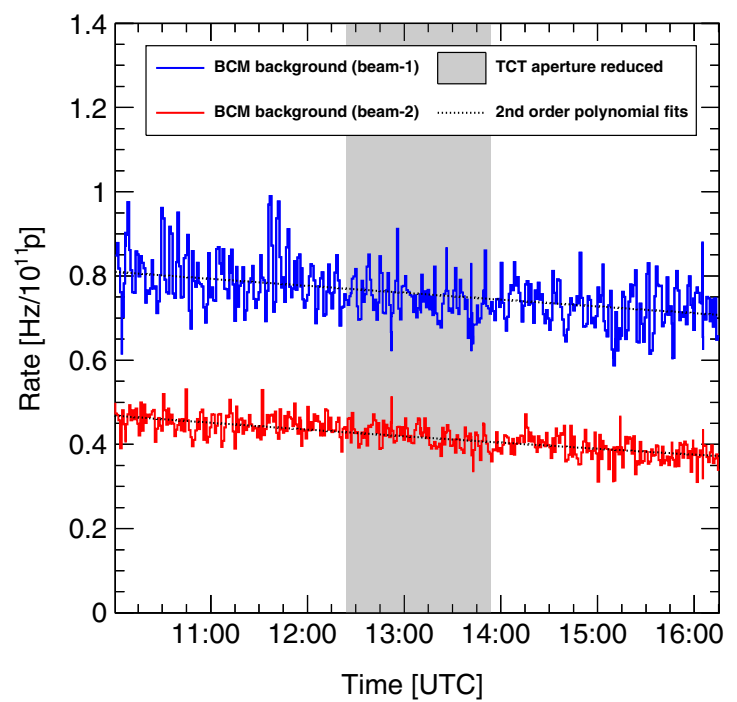

FIG. 7. B1 and B2 background rates measured by the ATLAS BCM before, during and after the period when the vertical TCTs were tightened by about $0.6 \sigma$. The polynomial fits take into account only the periods before and after the period of reduced aperture. The data are taken from Ref. [24]. The larger fluctuations of the B1 background are due to small oscillations of the beam screen [50] temperature on the side of the incoming B1. sensitivity of the test, if all the observed background would originate from losses on the TCTs. Assuming that the protons from elastic beam-gas scattering have a similar betatron amplitude distribution as tertiary halo, this sets an upper limit of about $10 \%$ to the relative background contribution from proton losses on the TCT. This test, by itself, cannot distinguish between elastic protons and tertiary halo, but since the latter is estimated from the lossmap test to be less than a percent, the $10 \%$ can be considered as an upper limit to the elastic scattering contribution.

The sensitivity of this test could be improved by closing also the horizontal TCT, and possibly by a larger amount, in order to increase the losses. But in order to do this in physics conditions a corresponding machine protection qualification is required.

\section{COMPARISON WITH SIMULATIONS}

Since the measurements described in Sec. IV relate proton losses and experimental background with unprecedentedly small uncertainties, they provide excellent data to compare with beam-background simulations. The latter follow a three-step approach, first proposed in Ref. [7], which conveniently divides the task into accelerator and detector parts such that the most appropriate simulation tools can be used for both. The accelerator simulations, which consist of first tracking the beam halo from the TCP to the TCT and then transporting the particle showers from the TCT to a virtual interface plane between the machine and the experiment are described in detail in Refs. [16,31]. The positions, four-momenta and types of particles crossing the interface plane serve as input for the detector simulations.

\section{A. Tracking simulations in LHC lattice}

In the first step of the accelerator simulations, the sixdimensional multiturn tracking code SIXTRACK [29,51-54] is used. It does a thin-lens element-by-element tracking through the magnetic lattice and when a proton encounters a collimator, a built-in scattering routine is invoked to simulate the proton-matter interactions. The tracking continues if the proton scatters back into the beam vacuum. The same simulation setup as in Ref. [29] is used. The starting conditions are halo protons with amplitudes large enough to hit a TCP. The diffusion of protons from the beam core [55] is not modeled, in order to keep the computing time feasible. At least $6 \times 10^{6}$ halo protons per configuration are tracked for 200 turns.

A proton is considered to be lost either when it undergoes an inelastic interaction inside a collimator or if it hits the aperture of any other element. The tracking in a collimator stops at the position of the inelastic interaction and no secondary particles are generated. The exception is single diffractive events, where the beam proton survives 


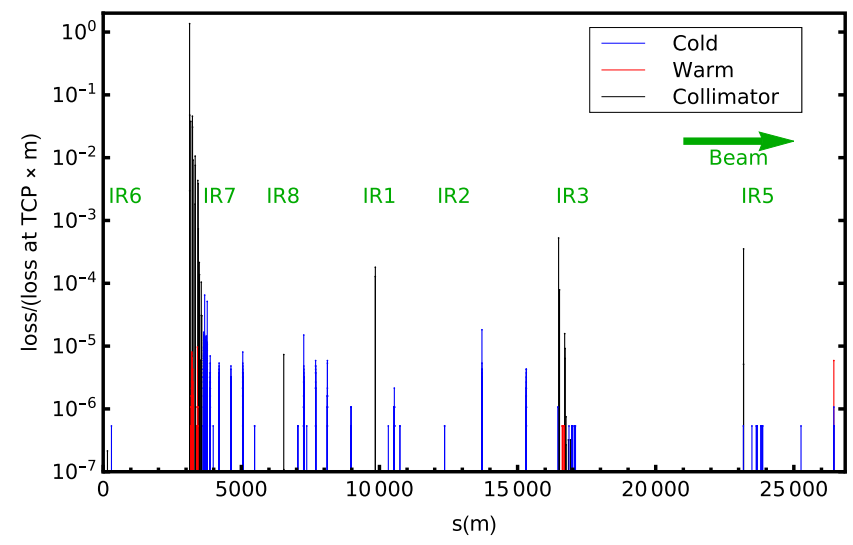

FIG. 8. B1 loss distribution at $\beta^{*}=40 \mathrm{~cm}$ around the LHC as simulated with SIXTRACK for an initial excitation in the vertical plane. The values correspond to rates summed over $0.1 \mathrm{~m}$ wide bins. The color indicates if the monitor is attached to a cold element (blue), a warm element (red), or a collimator (black).

and is tracked further. The SIXTRACK simulation output consists of the proton coordinates at all loss locations.

Figure 8 shows a typical result of the SIXTRACK-simulated losses around the ring, using the same settings as those used for the studies in Fig. 2. Like in similar studies at 3.5 TeV [29] a good qualitative agreement between SIXTRACK results and BLM measurements is seen. Quantitatively, the loss distributions shown in Figs. 2 and 8 are not strictly comparable, since the simulation shows only the loss positions of primary beam protons, while the BLM signals are produced by the

TABLE V. SIXTRACK predictions of the fraction of total losses that end up on the TCTs in IR1 for the machine configurations during the loss-map tests of this study: $\beta^{*}=80 \mathrm{~cm}$ with TCTs at $13.7 \sigma$ as in 2015 operation, and $\beta^{*}=40 \mathrm{~cm}$ with TCTs at $8.8 \sigma$, where the latter is also close to the 2016 configuration for physics operation. The presented values are the ratio of total losses on the horizontal and vertical TCTs with respect to all halo related losses around the machine.

\begin{tabular}{lcc}
\hline \hline Beam & $\beta^{*}=40 \mathrm{~cm}$ & $\beta^{*}=80 \mathrm{~cm}$ \\
\hline B1 & $3.6 \times 10^{-4}$ & $1.1 \times 10^{-5}$ \\
B2 & $1.4 \times 10^{-4}$ & $1.4 \times 10^{-5}$ \\
\hline \hline
\end{tabular}

showers which are created by the proton impacts. These distribute the energy loss over a larger distance, depending on the local geometry.

Figure 8 shows vertical losses of $\mathrm{B} 1$ at $\beta^{*}=40 \mathrm{~cm}$, but similar simulations have been performed for all cases relevant to this study. The obtained fractions of total simulated losses that end up on the TCTs are listed in Table V. These provide the normalization factors, i.e. are used to express the simulated background per proton lost on the TCP. The results at $\beta^{*}=40 \mathrm{~cm}$ correspond to what might be intuitively expected: the TCT losses are larger in B1, for which the path from IR7 to IR1 is shorter than for $\mathrm{B} 2$ and where fewer collimators are passed. This is not the case at $\beta^{*}=80 \mathrm{~cm}$, where the contributions from both beams are roughly equal due to differences in the phase advance between the IR7 TCSGs, the IR1 TCTs and other collimators in between: halo might pass a tight collimator at a location where betatron oscillations have a small transverse offset and be intercepted on a wider aperture at a position where the offset it large. For both beams, however, the TCT loss fraction is by about an order of magnitude larger at $\beta^{*}=40 \mathrm{~cm}$ than at $\beta^{*}=80 \mathrm{~cm}$. This difference arises from the much smaller TCT opening of $8.8 \sigma$ at $\beta^{*}=$ $40 \mathrm{~cm}$ as opposed to $13.7 \sigma$ at $\beta^{*}=80 \mathrm{~cm}$.

Even though the SIXTRACK results cannot be compared with the data in absolute terms, it is interesting to compare the ratio of TCT hits between different machine configurations with the ratio of measured background. Such comparisons, shown in Table VI, assume implicitly that the background rate is directly proportional to the TCT hit rate, so that unknown factors, in particular the number of observed background events per TCT hit, cancel out. This proportionality can, however, be violated by a dependence between impact depth and background production. In particular, deeper TCT impacts can be assumed to result in less shower leakage and hence lower background per TCT hit.

The measured values in Table VI indicate that the B1/B2 background ratio is about 2 at $\beta^{*}=40 \mathrm{~cm}$, while it is around 0.5 at $\beta^{*}=80 \mathrm{~cm}$. These observations agree with the SIXTRACK ratios which are derived from Table V. Consequently the measured $\beta^{*}=40 \mathrm{~cm} / \beta^{*}=80 \mathrm{~cm}$ is about 4 times larger for B1 than for B2. The fact that

TABLE VI. Ratios of measured background counts per TCP hit between various configurations, calculated from Table II, shown together with the corresponding ratios of simulated hits on the TCTs, taken from Table V. The average background over horizontal and vertical losses is considered. For the simulations, the sum of hits on the horizontal and vertical TCTs is used. For $\beta^{*}=40 \mathrm{~cm}$ the TCT setting of $8.8 \sigma$ is considered, which is closest to the 2016 operational configuration.

\begin{tabular}{lccc}
\hline \hline Ratio & Measured BCM & Measured fake jets & Simulated TCT impacts \\
\hline Beam 1/beam 2, $\beta^{*}=40 \mathrm{~cm}$ & $1.66 \pm 0.04$ & $1.9 \pm 0.1$ & 2.6 \\
Beam 1/beam 2, $\beta^{*}=80 \mathrm{~cm}$ & $0.55 \pm 0.03$ & $0.45 \pm 0.07$ & 0.71 \\
$\left(\beta^{*}=40 \mathrm{~cm}\right) /\left(\beta^{*}=80 \mathrm{~cm}\right)$, beam 1 & $19.8 \pm 0.9$ & $35 \pm 5$ & 32.5 \\
$\left(\beta^{*}=40 \mathrm{~cm}\right) /\left(\beta^{*}=80 \mathrm{~cm}\right)$, beam 2 & $6.6 \pm 0.2$ & $8.4 \pm 0.8$ & 9.9 \\
\hline \hline
\end{tabular}


background measurements and SIXTRACK agree on this asymmetry between the beams is a strong indication that the effects of different machine configurations are well modeled in the simulations.

Overall, the fake-jet ratios agree better with the simulations than the BCM ratios, especially for B1. This suggests that the assumption of direct proportionality between impact rate on the TCT and observed background is to some extent violated for the BCM background. Being very close to the beam line, the BCM is likely to be more sensitive to the proton impact distribution on the TCT, and in particular the number of impacts close to the TCT surface. Because the fake jets are dominated by highenergy muons at large radii, the TCT impact distribution is expected to be less important.

Despite the discrepancies, the overall agreement between simulation and measurement in Table VI is rather good and in all cases within a factor 2. This suggests that the fractional losses on the TCTs from SIXTRACK alone can be used to estimate, within a factor of a few, the beam-halo background in a future machine configuration if it is known in the present configuration. This method could be a relatively easy way to estimate whether the beam-halo background in a future, untested, configuration risks to become significant.

\section{B. TCT shower simulation}

The second simulation step is the shower propagation from the TCTs to the experiment, using the particle physics Monte Carlo simulation code FLUKA [56,57] as described in Refs. [16,31]. The FLUKA simulations use as starting conditions the TCT impacts from SIXTRACK to generate inelastic interactions, averaged over horizontal and vertical losses, from which the particle showers are propagated through the accelerator structures to a virtual interface plane, next to the experiment. This plane is defined to be between the quadrupole triplet, providing the final focus at the IP, and a fixed absorber (TAS), which is installed to protect the superconducting magnets from collision debris from the IP. This location, at $22.6 \mathrm{~m}$ from the IP, forms a natural boundary between the accelerator geometry model and that of the ATLAS experimental area.

A detailed 3D geometry of the about 130 m-long region between the interface plane and the TCTs, as implemented in FLUKA, is shown in Fig. 9. It includes the magnetic fields in the lattice between the TCT and the experiment. Because of symmetry, only one side of the experiment is implemented and used for the simulation of both beams. Contrary to most previous studies $[7,14,15]$, no variance reduction was applied so that all correlations within an event are preserved. The FLUKA simulations were done for both beams and $\beta^{*}$ settings.

Since the two background measurements by ATLAS are sensitive to very different radiation components, two sets of FLUKA simulations were performed. For the BCM studies,

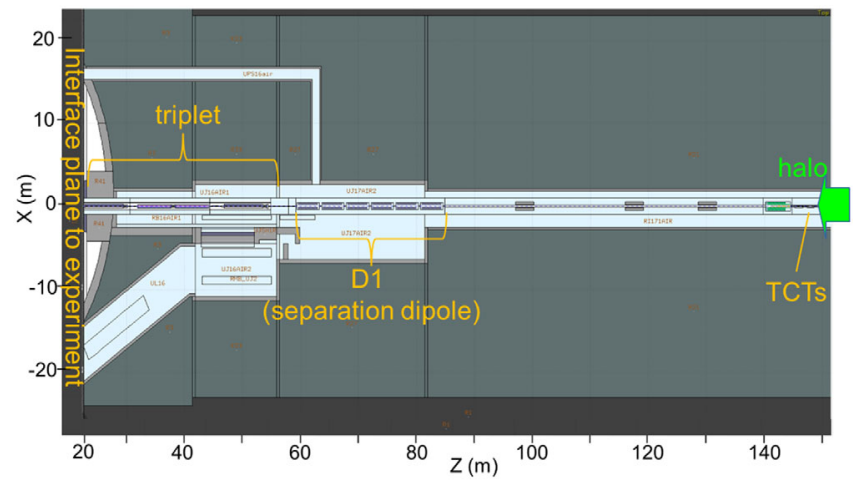

FIG. 9. Top view of the IR1 geometry implemented in FLUKA. The TCTs, which are intercepting the halo, are located at the right edge at about $145 \mathrm{~m}$ from the IP. The induced shower is simulated up to a virtual interface plane to the experiment, at the left edge of the figure at $22.6 \mathrm{~m}$ from the IP.

where low-energy particles are important, a $20 \mathrm{MeV}$ cutoff was used to produce a sample of $5 \times 10^{6}$ events. The fake jets are almost exclusively due to radiative energy losses of high-energy muons in the ATLAS calorimeters. In order to produce a sufficient number of the relatively rare events with large energy loss, another sample of $3 \times 10^{8}$ simulated events was produced, using a $20 \mathrm{GeV}$ transport cutoff. This value is chosen since the single-jet trigger with $12 \mathrm{GeV}$ threshold, used to select fake jets from the data, reaches full efficiency around $20 \mathrm{GeV}$ [24].

Examples of simulated particle distributions at the interface plane are shown in Figs. 10 and 11. Figure 10

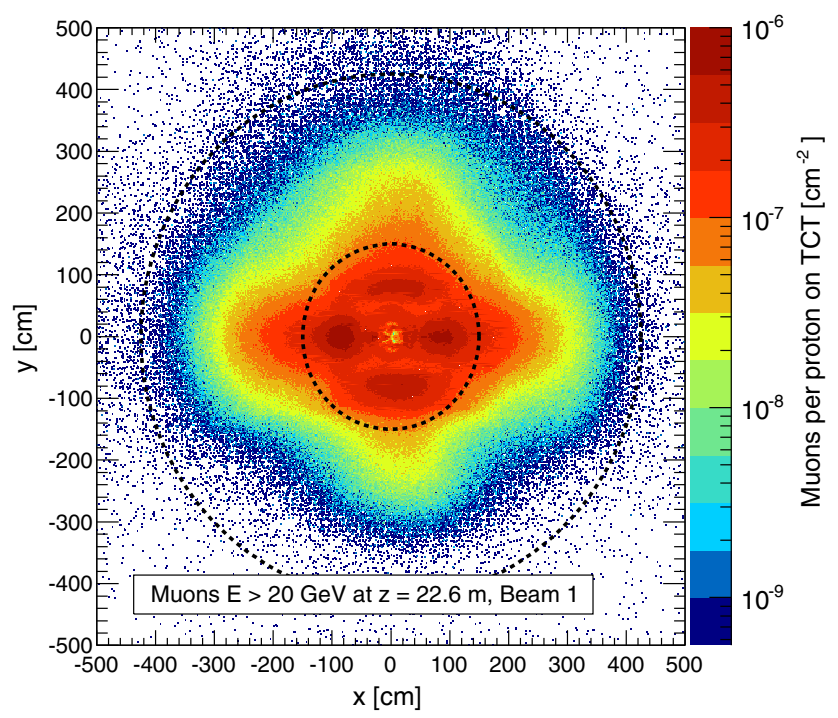

FIG. 10. Distributions of muons with an energy above $20 \mathrm{GeV}$ crossing the machine-detector interface plane, as simulated with FLUKA, for beam-halo losses on the TCTs for the 2015 machine with $\beta^{*}=80 \mathrm{~cm}$. The dashed circles indicate the inner and outer radii of the ATLAS barrel calorimeters. These serve only as rough guidelines, since the plot does not include the transport from the interface plane to the calorimeters. 


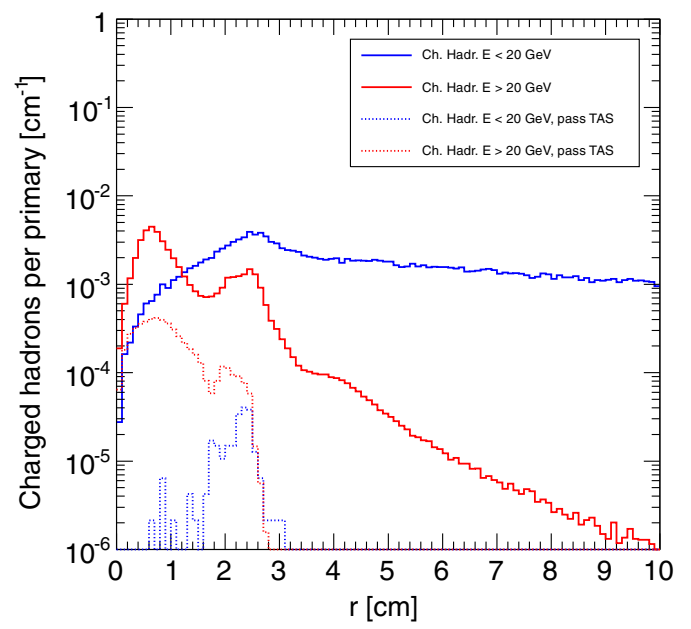

FIG. 11. Radial distributions of charged hadrons crossing the interface plane close to the beam line, as simulated with FLUKA for beam-halo losses on the TCTs assuming the 2015 machine optics with $\beta^{*}=80 \mathrm{~cm}$. The solid histograms show the totals in two energy ranges, while the dotted ones correspond to particles which, using a straight extrapolation of the trajectory, pass the TAS aperture but cross the beam-pipe wall upstream of the BCM detector. The histograms are normalized per radial unit in order to give a better impression of the number of particles at any given radius.

shows the distribution of high-energy muons, which are susceptible to produce fake jets in the ATLAS calorimeters. The azimuthal structure of the incident muon flux, especially the maxima in the horizontal and vertical planes, is due to bending of the muon trajectory in the D1 dipole and the focusing quadrupoles of the inner triplet. During the FLUKA transport from the interface plane to the calorimeters the muons will spread out due to scattering, energy loss and bending in the forward toroid magnet of ATLAS. Figure 11 shows the distribution of charged hadrons at the interface plane. The contribution of those particles for which a trajectory projection passes the $r=17 \mathrm{~mm}$ aperture of the TAS, but exceeds the $23.5 \mathrm{~mm}$ radius of the beam pipe before the first BCM station, is shown separately. Their particular relevance will be discussed in the next section.

\section{ATLAS detector simulation}

The third simulation step is the transport of the particles from the interface plane through ATLAS, while recording quantities that are needed to reconstruct the background observables. Also for these simulations, described in full detail in Ref. [24], FLuKA is used. The transport threshold is set to $100 \mathrm{keV}$ in order to include all particles possibly giving hits in the $\mathrm{BCM}$, which records a hit when the deposited energy exceeds $\sim 250 \mathrm{keV}$. This is about $1 / 3$ of the average energy loss of a minimum ionizing particle traversing the $1 \mathrm{~mm}$-thick diamond module at normal angle of incidence. Charged particles with an energy just exceeding $250 \mathrm{keV}$ will stop in the module, depositing all of their energy.

In order to reconstruct the $\mathrm{BCM}$ trigger signature, the times of all hits must be known with respect to the bunch passage at the IP. In the simulations, the time of flight is set to 0 at the impact point in the TCT and then rigorously propagated through the shower simulations. Analogous to the real data, a BCM background count is formed when an upstream and a downstream module have a hit in their corresponding trigger time windows, within the same event [24]. The time spread caused by the LHC bunch length of $7.5 \mathrm{~cm}$ is accounted for by smearing the time of each event correspondingly. In addition, each BCM hit is smeared by $600 \mathrm{ps}$ in order to model the instrumental time resolution of the BCM modules [24]. To match the threshold of the BCM modules in the simulations, particles with $E>250 \mathrm{keV}$ entering a BCM module were counted as hits.

Since FLUKA simulations are not compatible with full ATLAS data reconstruction, a customized and simplified method to estimate the rate of fake jets is developed in Refs. [24,44]: the energy deposited in an $(r, \phi, z)$ binning covering the calorimeters is analyzed for each event and energy deposition clusters exceeding $p_{\mathrm{T}}=12 \mathrm{GeV} / c$ are recorded as fake jets. To allow for the full turn-on of the ATLAS jet trigger, the simulated fake-jet rates are compared with data only above $p_{\mathrm{T}}=20 \mathrm{GeV} / c$ [24].

The results of the complete simulation chain, from the impacts on the TCPs to the simulated background rates in ATLAS, are compared with the ATLAS measurements in Table VII. These results are taken from Ref. [24], but the simulated rates have been rescaled to correspond to the most recent SIXTRACK simulations. In all cases the rates are normalized to one proton lost on the TCPs by using the TCT loss fractions in Table V, which correspond to the configuration during the loss-map tests. For B1 at $\beta^{*}=$ $80 \mathrm{~cm}$ the simulations and measurements are in very good agreement, while an underestimation by a factor of $\sim 2$ is found for the $\mathrm{B} 2$ simulations. At $\beta^{*}=40 \mathrm{~cm}$ the agreement is not as good for B1, where the simulated rates are more than a factor of 3 below the measurements. Even the largest ratio of $\sim 3.5$, however, can still be considered a good agreement, when considering the complexity of the threestep simulation chain and the fact that the simulated losses around the ring span over many orders of magnitude. In particular, the SIXTRACK simulations assume a perfect LHC geometry and optics, while it has been shown that realistic imperfections can increase the rates on the TCT by a factor of 2-3 [29]. The $\mathrm{B} 2$ rates, and those for $\mathrm{B} 1$ at $\beta^{*}=80 \mathrm{~cm}$, are consistent within this uncertainty but for the $\mathrm{B} 1$ rates at $\beta^{*}=40 \mathrm{~cm}$ the agreement is slightly outside this estimated error margin.

In order to further investigate the largest discrepancy, found in $\mathrm{B} 1$ at $\beta^{*}=40 \mathrm{~cm}$, the dependence on the TCT impact distribution was studied. While the distribution of the transverse depth $(d)$ of the inelastic interactions inside 
TABLE VII. Comparison of measured background rates with simulations, normalized to the number of protons lost from the bunch. The data for the BCM background are the same as in Table II. The errors are statistical only. The ATLAS data and simulations are taken from Ref. [24], but the simulation results have been rescaled to the most recent SIXTRACK results in Table V.

\begin{tabular}{|c|c|c|c|c|}
\hline \multicolumn{5}{|c|}{$\beta^{*}=80 \mathrm{~cm}$, TCT at $13.7 \sigma$} \\
\hline Background observable & Beam & Data & Simulation & Ratio \\
\hline BCM background & 1 & $(5.2 \pm 0.2) \times 10^{-9}$ & $(5.4 \pm 0.1) \times 10^{-9}$ & $0.97 \pm 0.04$ \\
\hline Fake jets $(>20 \mathrm{GeV})$ & 1 & $(2.5 \pm 0.4) \times 10^{-10}$ & $(2.43 \pm 0.04) \times 10^{-10}$ & $1.0 \pm 0.2$ \\
\hline BCM background & 2 & $(9.4 \pm 0.3) \times 10^{-9}$ & $(4.5 \pm 0.1) \times 10^{-9}$ & $2.09 \pm 0.09$ \\
\hline Fake jets $(>20 \mathrm{GeV})$ & 2 & $(5.4 \pm 0.7) \times 10^{-10}$ & $(2.33 \pm 0.05) \times 10^{-10}$ & $2.3 \pm 0.3$ \\
\hline \multicolumn{5}{|c|}{$\beta^{*}=40 \mathrm{~cm}$, TCT at $8.8 \sigma$} \\
\hline Background observable & Beam & Data & Simulation & Ratio \\
\hline BCM background & 1 & $(10.3 \pm 0.2) \times 10^{-8}$ & $(2.83 \pm 0.16) \times 10^{-8}$ & $3.6 \pm 0.2$ \\
\hline Fake jets $(>20 \mathrm{GeV})$ & 1 & $(8.8 \pm 0.5) \times 10^{-9}$ & $(2.93 \pm 0.08) \times 10^{-9}$ & $3.0 \pm 0.2$ \\
\hline BCM background & 2 & $(6.2 \pm 0.1) \times 10^{-8}$ & $(5.2 \pm 0.1) \times 10^{-8}$ & $1.19 \pm 0.04$ \\
\hline Fake jets $(>20 \mathrm{GeV})$ & 2 & $(4.1 \pm 0.3) \times 10^{-9}$ & $(2.46 \pm 0.05) \times 10^{-9}$ & $1.7 \pm 0.1$ \\
\hline
\end{tabular}

the TCT jaws, obtained from the SIXTRACK simulations, is very similar at $\beta^{*}=40 \mathrm{~cm}$ and $\beta^{*}=80 \mathrm{~cm}$ for $\mathrm{B} 2$, a significant difference is seen for B1. This is shown in the leftmost plot in Fig. 12. Table VIII lists, for each configuration, the fraction of very shallow impact depths, i.e. those corresponding to the leftmost bin in Fig. 12 . At $\beta^{*}=80 \mathrm{~cm}$, for B1, most impacts are at rather shallow depth, and $35 \%$ have $d<50 \mu \mathrm{m}$, while at $\beta^{*}=40 \mathrm{~cm}$ there is only a minor excess of $5.8 \%$ at $d<50 \mu \mathrm{m}$, followed by a long, almost flat, tail. This suggests that a larger fraction of the showers will leak back into the beam line at $\beta^{*}=80 \mathrm{~cm}$. The middle plot in Fig. 12 shows that the BCM background events, reconstructed in the complete FLUKA simulation, originate indeed from very shallow impacts-about 90\%-97\% of them are due to protons lost at $d<50 \mu \mathrm{m}$. For fake jets, on the other hand, typically $50 \%$ come from impacts at $d>50 \mu \mathrm{m}$, as can be seen from the rightmost plot. Hence, a possible explanation as to why the agreement between simulations and measurement is not as good for B1 at $\beta^{*}=40 \mathrm{~cm}$ as in the other cases, could be an inaccuracy of the simulated impact depth $d$. This could possibly be due to ignoring the effect of imperfections in the simulations. Imperfections are
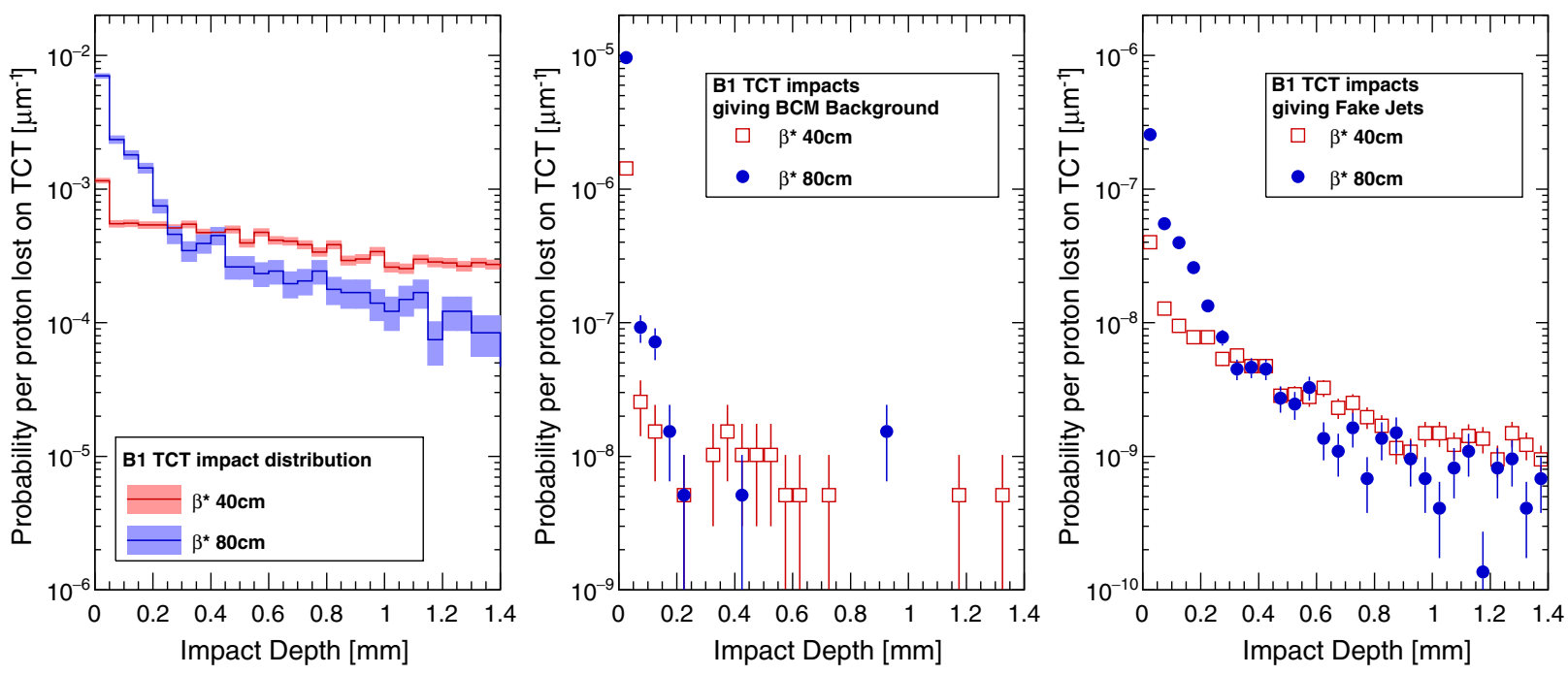

FIG. 12. Distribution of the transverse depth of the inelastic interactions inside the jaws of the TCTs in B1 at $\beta^{*}=40 \mathrm{~cm}$ and $\beta^{*}=80 \mathrm{~cm}$ for a superposition of horizontal and vertical losses in IR7. The probabilities, obtained from the simulations, are averaged over $50 \mu \mathrm{m}$ wide bins. The TCT apertures are $8.8 \sigma$ and $13.7 \sigma$ at $\beta^{*}=40 \mathrm{~cm}$ and $\beta^{*}=80 \mathrm{~cm}$, respectively. The hits in the horizontal and vertical TCTs are summed. The left plots show the distribution of all impacts. The middle and right plots show only those impacts that have resulted in a BCM background signature or a fake jet, respectively. 
TABLE VIII. SIXTRACK predictions of the fraction of impacts at $d<50 \mu \mathrm{m}$ in the TCT jaws. The hits in horizontal and vertical TCTs are summed. The values correspond to the first bins in the left plots of Fig. 12.

\begin{tabular}{lcc}
\hline \hline Beam & $\beta^{*}=40 \mathrm{~cm}$ & $\beta^{*}=80 \mathrm{~cm}$ \\
\hline B1 & 0.058 & 0.35 \\
B2 & 0.28 & 0.24 \\
\hline \hline
\end{tabular}

known to affect the results by up to a factor of 3 [29] and their effect should be studied for both B1 and B2.

In this context it is interesting to return to the particles passing the TAS aperture, shown by the dotted lines in Fig. 11. The FluKA results reveal that these particles give $40 \%$ of the simulated BCM triggers. Further $25 \%$ are due to photons passing the TAS aperture. Thus the simulations suggest that about $2 / 3$ of the background seen by the BCM is due to particles not interacting with the TAS, but showering on the beam pipe inside ATLAS. Furthermore, all charged hadrons passing the TAS and leading to BCM triggers in the simulations have energies above $100 \mathrm{GeV}$ and a majority even several $\mathrm{TeV}$. These are exactly the expected characteristics of particles scattered back into the beam vacuum from events at very shallow depth. This finding also provides justification for the $20 \mathrm{MeV}$ cutoff used in the LHC simulations, although the BCM has some sensitivity to particles with much lower energies. In fact, the simulated $\mathrm{BCM}$ background rate would be reduced by only $8 \%$ even if the sample with $20 \mathrm{GeV}$ transport cutoff were used [24].

Figure 13 compares the shapes of simulated and measured distributions of the fake jets at $\beta^{*}=40 \mathrm{~cm}$ for B1. It is assumed that the shapes of the distributions are independent of the rate or impact depth distribution on the TCT.

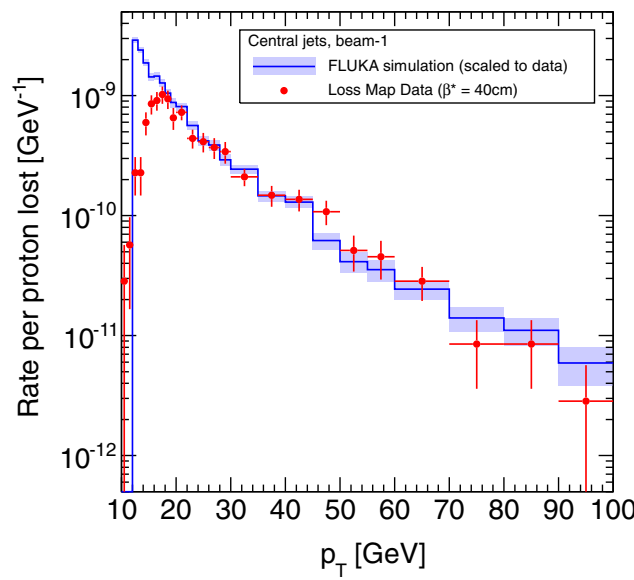

Therefore, in order to focus the comparison on the shapes only, the simulation results have been scaled to the data by using the ratios of data to simulations given in Table VII. The agreement on an absolute scale can be appreciated from Table VII. The upper plot compares the distributions in apparent transverse momentum, $p_{\mathrm{T}}$. The effect of the ATLAS jet trigger turn-on is visible below $20 \mathrm{GeV}$, where the data drops down, while the simulated rate continues to rise towards lower transverse momenta. The bottom plot shows the distributions in azimuthal angle $\phi$, defined with respect to the horizontal axis, which points towards the center of the LHC ring. The highest fake-jets rates are found around $\phi=0$ and $\phi=\pi$, i.e. in the horizontal plane. These maxima are caused by muons of opposite charge being separated and swept out in opposite directions by the separation dipole D1 between the TCT and the experiment [16]. The minor peaks at $\pm \pi / 2$ are due to bending in the quadrupoles of the inner triplet, providing the final focus at the IP. The simulation results reproduce the shapes of both distributions within statistical uncertainties.

\section{CONCLUSIONS}

The first isolated measurements of beam-halo background in the experimental detectors at the LHC have been presented. The results are based on dedicated loss-map tests where low-intensity beams have been excited in order to induce beam losses on the primary TCP collimators. A small fraction of the protons leaks to the tertiary TCT collimators in front of the experiments and induces showers which are detected as machine-induced background. In these particular tests, the ATLAS experiment was recording background data, contrary to the routinely performed loss maps at the LHC. With the low beam intensity, but artificially provoked

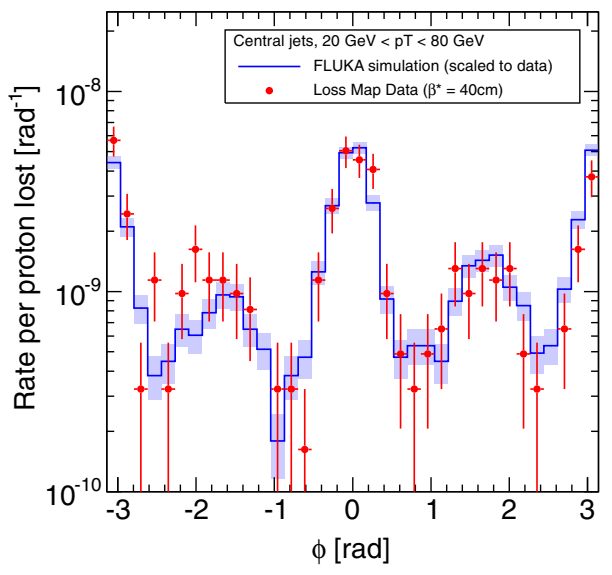

FIG. 13. Comparison of simulated $p_{\mathrm{T}}$ and azimuthal distributions of fake jets with data for B1 losses at $\beta^{*}=40 \mathrm{~cm}$ and a TCT aperture of $8.8 \sigma$. In both plots only jets in the central barrel calorimeters are considered. For the $\phi$ distributions an additional requirement of $p_{\mathrm{T}}>20 \mathrm{GeV}$ has been applied in order to stay in the region where the ATLAS jet trigger is fully efficient. The azimuthal angle $(\phi)$ is measured with respect to the $\mathrm{x}$ axis, which in the ATLAS coordinate system points towards the center of the LHC ring. Only the statistical uncertainties of the simulations and the data are indicated. The data and simulations are taken from [24] but have been rebinned into a wider structure at large $p_{\mathrm{T}}$ where statistics is poor. In order to facilitate the shape comparison, the simulation results have been scaled to the data using the ratios given in Table VII. 
short and intense loss spikes, the showers from the TCTs were the by far dominating source of background in the experiments. The results show that both the measured losses on the TCTs, as well as the observed background in ATLAS, as a function of the TCT aperture, can be fitted with a simple exponential.

The pure halo-related backgrounds measured in these tests are scaled to typical loss rates on the TCPs during standard operation, which, from two independent analyses, were found to be a few $10^{5}$ protons per second per bunch during the first hour of collisions. The fraction of background coming from beam halo was estimated to be at the per-mil level of the total background in 2015 and at the percent level in 2016. This confirms the indication obtained in previous studies $[21,22]$ that the machine-induced background in the ATLAS experiment is dominated by local inelastic beam-gas interactions. A second test, in which the apertures of the vertical TCTs were slightly reduced during a high-intensity physics fill, supports this hypothesis, as no visible change in background was observed. This shows also that the background from elastic beam-gas scattering sending protons on the TCTs is negligible. The simulation studies presented in Ref. [44] indicate that the beam-gas interactions, which contribute to the background in ATLAS, take place within $\sim 500 \mathrm{~m}$ of the experiment. Dedicated studies, injecting small amounts of gas locally into the beam vacuum, were undertaken during LHC run 2 in order to experimentally determine the sensitivity to beam-gas events as a function of distance from the experiment. The analysis of the data recorded during these tests is ongoing and is expected to provide verification for the regions where vacuum optimization has the largest impact on the background.

The results were used to estimate the effect on background from smaller TCT apertures. For all realistic TCT settings, which respect the hierarchy of the multistage collimation system, the beam-halo background is so small that it does not impose a constraint on the TCT aperture. This result has significant implications for the future optimization of the performance of the LHC, as well as the future high-luminosity LHC [58], since the TCT setting is directly connected to the aperture that can be protected and hence the achievable $\beta^{*}$. A tighter TCT allows reducing $\beta^{*}$, which results in a corresponding increase in luminosity. The fact that the TCT setting is not constrained by beamhalo background does, however, not mean that it can be arbitrarily tight. Other constraints from protection of the triplet magnets and the TCT itself, in particular during accidental losses, are the present limitation at the LHC for further reductions of $\beta^{*}$ [34,59], although they have been relaxed through the use of a specially matched optics [3].

The measurements have been compared with detailed three-step simulations. The first simulation step consists of tracking halo protons, with the SIXTRACK code, to estimate the impacts on the TCTs. In the second step FLUKA is used to generate inelastic interactions of protons in the TCT material and transporting the particle showers to a virtual interface plane close to the experiment. In the third and final step the particles recorded at the interface plane are transported with FLUKA through the ATLAS geometry and hit rates and energy depositions are recorded event by event. The simulation results are normalized with the bunch intensity loss, which during the loss-map test are almost entirely due to losses on the TCPs. Taking into account systematic uncertainties, in particular possible machine imperfections which are not considered in the simulations, a good quantitative agreement with the measured background is obtained. Features like transverse momentum and azimuthal distributions of fake jets are accurately reproduced.

Furthermore, it is shown that if the background is known for one configuration, SIXTRACK simulations of the transfer from the TCPs to the TCTs could be used to predict the background for other configurations of TCT settings and optics, with an accuracy of about a factor of a few, as long as the TCT impact distribution does not change significantly.

These results demonstrate that the beam-halo background at the LHC experiments is well understood and that it is not in any way limiting the present operational performance of the LHC, nor does it seem to be a limiting factor of performance optimization in the foreseeable future.

\section{APPENDIX: ABBREVIATIONS}

We summarize, in alphabetical order, all abbreviations used throughout the paper: ADT-transverse damper, used also to excite the beam to provoke losses; ATLAS-a general-purpose particle detector experiment located in IR1 of the LHC; B1, B2-the two counterrotating beams in the LHC; BCM-beam condition monitor, detector used to measure near-beam backgrounds in ATLAS; BCT-beam current transformer, used to measure beam intensity; BLM —beam loss monitor; CMS - a general-purpose particle detector experiment located in IR5 of the LHC; IPinteraction point; IR-insertion region [the LHC has eight IRs (see Fig. 1)]; LHC-large hadron collider [1,2]; TASfixed absorber placed close to the experimental detector; TCDQ-large absorber used for beam dump protection in IR6; TCLA — active absorber located in IR7 or IR3; TCPprimary collimator located in IR7 or IR3; TCSG-secondary collimator located in IR7 or IR3; TCSP—secondary collimator used for beam dump protection in IR6; TCTtertiary collimator located in the experimental insertions; and TCTH, TCTV-horizontal $(\mathrm{H})$ or vertical $(\mathrm{V})$ tertiary collimator located in the experimental insertions.

[1] LHC design report v.1: The LHC main ring, edited by O. S. Brüning, P. Collier, P. Lebrun, S. Myers, R. Ostojic, J. Poole, and P. Proudlock, Report No. CERN-2004-003-V1, 2004. 
[2] L. Evans and P. Bryant, LHC machine, J. Instrum. 3, S08001 (2008).

[3] R. Bruce, C. Bracco, R. De Maria, M. Giovannozzi, A. Mereghetti, D. Mirarchi, S. Redaelli, E. Quaranta, and B. Salvachua, Reaching record-low $\beta^{*}$ at the CERN Large Hadron Collider using a novel scheme of collimator settings and optics, Nucl. Instrum. Methods Phys. Res., Sect. A 848, 19 (2017).

[4] $\beta^{*}$ is the optical $\beta$-function at the IP.

[5] ATLAS Collaboration, The ATLAS experiment at the CERN Large Hadron Collider, J. Instrum. 3, S08003 (2008).

[6] CMS Collaboration, The CMS experiment at the CERN LHC, J. Instrum. 3, S08004 (2008).

[7] A. I. Drozhdin, M. Huhtinen, and N. V. Mokhov, Accelerator related background in the CMS detector at LHC, Nucl. Instrum. Methods Phys. Res., Sect. A 381, 531 (1996).

[8] Proceedings of the workshop on LHC backgrounds, CERN, Geneva, 1996, edited by K. M. Potter, Report No. CERN-2009-003, 1996.

[9] N. V. Mokhov, Comparison of backgrounds in detectors for LHC, NLC and $\mu^{+} \mu^{-}$colliders, Nucl. Phys. B, Proc. Suppl. 51, 210 (1996).

[10] I. Azhgirey, I. Baishev, V. Talanov, and K. M. Potter, Machine induced background in the high luminosity experimental insertion of the LHC project, in Proceedings of the 9th European Particle Accelerator Conference, Lucerne, 2004 (EPS-AG, Lucerne, 2004), p. 755.

[11] V. Talanov, Estimation of the machine induced background for the commissioning period with tertiary collimators in the IR1 of the LHC, LHC Project Note 371, CERN, 2005.

[12] G. Corti and V. Talanov, Aspects of machine induced background in the LHC experiments, LHC Project Workshop-Chamonix XV (CERN, Geneva, Switzerland, 2006), p. 178.

[13] Proceedings of the LHC workshop on experimental conditions and beam-induced detector backgrounds, CERN, Geneva, Switzerland, 2008, edited by R. Alemany-Fernandez, H. Burkhardt, M. Ferro-Luzzi, M. Lamont, A. Macpherson, and S. Redaelli et al., Report No. CERN-2009-003, 2009.

[14] N. V. Mokhov and T. Weiler, Machine-induced backgrounds: Their origin and loads on ATLAS/CMS, Report No. Fermilab-Conf-08-147-APC, 2018.

[15] A. I. Drozhdin, N. V. Mokhov, and S. I. Striganov, Beam losses and background loads on collider detectors due to beam-gas interactions in the LHC, in Proceedings of the 23rd Particle Accelerator Conference, Vancouver, Canada, 2009 (IEEE, Piscataway, NJ, 2009), pp. 2549-2551.

[16] R. Bruce et al., Sources of machine-induced background in the ATLAS and CMS detectors at the CERN Large Hadron Collider, Nucl. Instrum. Methods Phys. Res., Sect. A 729, 825 (2013).

[17] ATLAS Collaboration, Search for new phenomena with the monojet and missing transverse momentum signature using the ATLAS detector in $\sqrt{s}=7 \mathrm{TeV}$ proton-proton collisions, Phys. Lett. B 705, 294 (2011).

[18] ATLAS Collaboration, Search for long-lived stopped $r$-hadrons decaying out of time with $p p$ collisions using the ATLAS detector, Phys. Rev. D 88, 112003 (2013).

[19] ATLAS Collaboration, Search for pair-produced long-lived neutral particles decaying to jets in the ATLAS hadronic calorimeter in $p p$ collisions at $\sqrt{s}=8 \mathrm{TeV}$, Phys. Lett. B 743, 15 (2015).

[20] The ATLAS Collaboration, Search for dark matter and other new phenomena in events with an energetic jet and large missing transverse momentum using the ATLAS detector, J. High Energy Phys. 01 (2018) 126.

[21] ATLAS Collaboration, Characterisation and mitigation of beam-induced backgrounds observed in the ATLAS detector during the 2011 proton-proton run, J. Instrum. 8, P07004 (2013).

[22] ATLAS Collaboration, Beam-induced and cosmic-ray backgrounds observed in the ATLAS detector during the LHC 2012 proton-proton running period, J. Instrum. 11, P05013 (2016).

[23] R. Bruce, H. Garcia, P. Hermes, R. Kwee-Hinzmann, A. Mereghetti, D. Mirarchi, E. Quaranta, S. Redaelli, and A. Valloni, Collimation with tighter TCTs at $\beta^{*}=40 \mathrm{~cm}$, Report No. CERN-ACC-NOTE-2015-0036, 2015.

[24] ATLAS Collaboration, Beam backgrounds in the ATLAS detector during LHC loss-map tests at $\beta^{*}=40 \mathrm{~cm}$ and $\beta^{*}=80 \mathrm{~cm}$ at $E_{\text {beam }}=6.5 \mathrm{TeV}$, ATLAS PUB Note No. ATL-DAPR-PUB-2017-001, 2017.

[25] R.W. Assmann, Collimators and beam absorbers for cleaning and machine protection, in Proceedings of the LHC Project Workshop-Chamonix XIV, Chamonix, France (CERN, Geneva, Switzerland, 2005), p. 261.

[26] G. Robert-Demolaize, Design and performance optimization of the LHC collimation system, Ph.D. thesis, Universite Joseph Fourier, Grenoble, 2006.

[27] R. W. Assmann et al., The final collimation system for the LHC, in Proceedings of the 10th European Particle Accelerator Conference, Edinburgh, Scotland, 2006 (EPS-AG, Edinburgh, Scotland, 2006), p. 986.

[28] C. Bracco, Commissioning scenarios and tests for the LHC collimation system, Ph.D. thesis, EPFL Lausanne, 2008.

[29] R. Bruce et al., Simulations and measurements of beam loss patterns at the CERN Large Hadron Collider, Phys. Rev. ST Accel. Beams 17, 081004 (2014).

[30] G. Valentino et al., Final implementation, commissioning, and performance of embedded collimator beam position monitors in the large hadron collider, Phys. Rev. Accel. Beams 20, 081002 (2017).

[31] R. Kwee-Hinzmann, G. Bregliozzi, R. Bruce, F. Cerutti, L. S. Esposito, S. M. Gibson, A. Lechner, H. Garcia Morales, and C. Yin Vallgren, Machine-induced background simulation studies for LHC run 1, run 2 and HLLHC, Report No. CERN-ACC-2017-0025, 2017.

[32] R. Kwee-Hinzmann, G. Bregliozzi, R. Bruce, F. Cerutti, L. S. Esposito, S. M. Gibson, R. Kersevan, A. Lechner, and N. V. Shetty, First beam background simulation studies at IR1 for high luminosity LHC, in Proceedings of the 5th International Particle Accelerator Conference, Dresden, Germany (JACoW, Geneva, Switzerland, 2014), p. 1074, http://inspirehep.net/record/1311376.

[33] R. Kwee-Hinzmann, R. Bruce, F. Cerutti, L. S. Esposito, S. M. Gibson, and A. Lechner, Beam induced background studies in IR1 with the new HL-LHC layout, in Proceedings of the 6th International Particle Accelerator 
Conference, Richmond, VA (JaCoW, Geneva, Switzerland, 2015), p. 2184, http://inspirehep.net/record/1395314?ln=en.

[34] R. Bruce, R. W. Assmann, and S. Redaelli, Calculations of safe collimator settings and $\beta^{*}$ at the CERN Large Hadron Collider, Phys. Rev. ST Accel. Beams 18, 061001 (2015).

[35] D. Wollmann et al., First cleaning with LHC collimators, in Proceedings of the International Particle Accelerator Conference, Kyoto, Japan (JACoW, Geneva, Switzerland, 2010), p. 1237, http://inspirehep.net/record/1358439.

[36] G. Valentino, R. Aßmann, R. Bruce, S. Redaelli, A. Rossi, N. Sammut, and D. Wollmann, Semiautomatic beam-based LHC collimator alignment, Phys. Rev. ST Accel. Beams 15, 051002 (2012).

[37] B. Salvachua et al., LHC collimation cleaning and operation outlook, in Proceedings of the 2012 LHC beam operation workshop, Evian, France (CERN, Geneva, Switzerland, 2012).

[38] B. Salvachua et al., Cleaning performance of the LHC collimation system up to $4 \mathrm{TeV}$, in Proceedings of the 4th International Particle Accelerator Conference, IPAC2013, Shanghai, China, 2013 (JACoW, Geneva, Switzerland, 2013), p. 1002, http://inspirehep.net/record/1241922.

[39] E. B. Holzer et al., Beam loss monitoring system for the LHC, IEEE Nucl. Sci. Symp. Conf. Record 2, 1052 (2005).

[40] E. B. Holzer et al., Development, production and testing of 4500 beam loss monitors, in Proceedings of the 11th European Particle Accelerator Conference, Genoa, 2008 (EPS-AG, Genoa, Italy, 2008), p. 1134.

[41] The recorded value is the largest running sum anywhere in the $1 \mathrm{~s}$ interval.

[42] W. Hofle, R. Assmann, S. Redaelli, R. Schmidt, D. Valuch, D. Wollmann, and M. Zerlauth, Controlled transverse blow-up of high-energy proton beams for aperture measurements and loss maps, in Proceedings of the $3 \mathrm{rd}$ International Particle Accelerator Conference, New Orleans, LA, 2012 (IEEE, Piscataway, NJ, 2012), p. 4059.

[43] V. Cindro et al., The ATLAS beam conditions monitor, J. Instrum. 3, P02004 (2008).

[44] ATLAS Collaboration, Comparison of simulated and observed LHC beam backgrounds in the ATLAS experiment at $\mathrm{E}_{\text {beam }}=4 \mathrm{TeV}$, J. Instrum. 13, P12006 (2018).

[45] Since this background test was done in parallel with a standard qualification test, it was necessary to achieve the standard orbit, which requires collisions.

[46] The collimator settings are usually expressed as the half gap in units of the local betatron beam size $\sigma$, assuming a nominal normalized emittance of $3.5 \mu \mathrm{m}$.
[47] B. Salvachua, D. Mirarchi, M. Pojer, S. Redaelli, R. Rossi, G. Valentino, and M. Wyszynski, Decomposition of beam losses at LHC, in Proceedings of the 8th International Particle Accelerator Conference, Copenhagen, Denmark (JACoW, Geneva, Switzerland, 2017), p. 88, http:// inspirehep.net/record/1643447.

[48] S. Redaelli and B. Salvachua, Analysis of 2016 beam losses at the LHC, in Proceedings of the 7th Evian workshop, Evian, France (CERN, Geneva, Switzerland, 2016).

[49] F. Antoniou et al., Can we predict luminosity?, in Proceedings of the 7th Evian workshop, Evian, France (CERN, Geneva, Switzerland, 2016).

[50] P. Cruikshank et al., Mechanical design aspects of the LHC beam screen, in Proceedings of the Particle Accelerator Conference, Vancouver, BC, Canada, 1997 (IEEE, New York, 1997), Vol. 3, pp. 3586-3588.

[51] F. Schmidt, SixTrack User's Reference Manual, Report No. CERN/SL/94-56-AP, 1994.

[52] G. Robert-Demolaize, R. Assmann, S. Redaelli, and F. Schmidt, A new version of SIXTRACK with collimation and aperture interface, in Proceedings of the 21st Particle Accelerator Conference, Knoxville, TN, 2005 (IEEE, Piscataway, NJ, 2005), p. 4084.

[53] C. Tambasco, An improved scattering routine for collimation tracking studies at LHC, Master's thesis, Università di Roma, Italy, 2014.

[54] SIXTRACK web site, http://sixtrack.web.cern.ch/SixTrack/.

[55] G. Valentino, R. Aßmann, R. Bruce, F. Burkart, V. Previtali, S. Redaelli, B. Salvachua, G. Stancari, and A. Valishev, Beam diffusion measurements using collimator scans in the LHC, Phys. Rev. ST Accel. Beams 16, 021003 (2013).

[56] A. Ferrari, P. R. Sala, A. Fassò, and J. Ranft, FLUKA: A multiparticle transport code, CERN Report No. CERN2005-10, 2005.

[57] T. T. Bohlen, F. Cerutti, M. P. W. Chin, A. Fassò, A. Ferrari, P. G. Ortega, A. Mairani, P. R. Sala, G. Smirnov, and V. Vlachoudis, The FLUKA code: Developments and challenges for high energy and medical applications, Nucl. Data Sheets 120, 211 (2014).

[58] High-luminosity Large Hadron Collider (HL-LHC): Technical design report V. 0.1, CERN Yellow Reports: Monographs, edited by G. Apollinari, I. Bejar Alonso, O. Bruning, P. Fessia, M. Lamont, L. Rossi, and L. Tavian et al., CERN Report No. CERN-2017-007-M, 2017.

[59] E. Quaranta, A. Bertarelli, R. Bruce, F. Carra, F. Cerutti, A. Lechner, S. Redaelli, E. Skordis, and P. Gradassi, Modeling of beam-induced damage of the LHC tertiary collimators, Phys. Rev. Accel. Beams 20, 091002 (2017). 